Bull. Soc. math. France

131 (3), 2003, p. $307-358$

\title{
SCHÉMAS EN GROUPES ET IMMEUBLES DES GROUPES EXCEPTIONNELS SUR UN CORPS LOCAL. PREMIÈRE PARTIE : LE GROUPE $G_{2}$
}

\author{
Par Wee Teck Gan \& Jiu-Kang Yu
}

\begin{abstract}
RÉSUmÉ. - Nous obtenons une version explicite de la théorie de Bruhat-Tits pour les groupes exceptionnels de type $G_{2}$ sur un corps local. Nous décrivons chaque construction concrètement en termes de réseaux : l'immeuble, les appartements, la structure simpliciale, les schémas en groupes associés. Les appendices traitent de l'analogie avec les espaces symétriques réels et des espaces symétriques associés à $G_{2}$ réel et complexe.

Abstract (Group Schemes and Buildings of Exceptional Groups over a Local Field. First Part : the Group $G_{2}$ )

We give an explicit Bruhat-Tits theory for the exceptional group of type $G_{2}$ over a local field. We describe every construct concretely in terms of lattices: the building, the apartments, the simplicial structure, and the associated group schemes. The appendices discuss analogy with symmetric spaces and the symmetric space of the real or complex $G_{2}$.
\end{abstract}

\section{Contents}

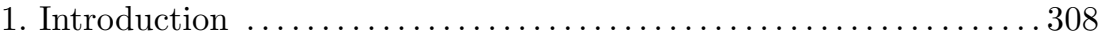

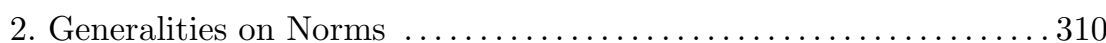

Texte reçu le 26 octobre 2001, accepté le 29 novembre 2001

Wee Teck Gan, Mathematics Department, Princeton University, Princeton, NJ 08544 (USA) E-mail : wtgan@math.princeton.edu - Url : http://www.math.princeton.edu/ ${ }^{\sim}$ wtgan JiU-Kang YU, Mathematics Department, University of Maryland, College Park, MD 20742 (USA) - E-mail : yu@math.umd.edu - Url : http://www.math.umd.edu/ yu Classification mathématique par sujets (2000). — MSC2000 : 11E95, 14L15, 14L40, 17A75, 22E50, 20G20, 20G25.

Mots clefs. - Schéma en groupes, théorie de Bruhat-Tits, immeuble, groupe exceptionnel. J.-K. Yu was partially supported by grant DMS 9801633 from NSF, USA. 
3. A General Formalism ...................................... 314

4. The Buildings of Classical Groups ...................... 317

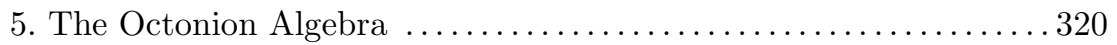

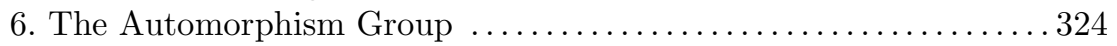

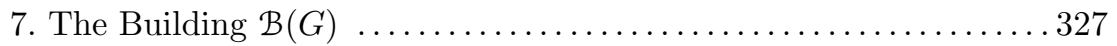

8. The Structure of Apartments . . . . . . . . . . . . . . . . 329

9. Simplicial Structure and Parahoric Subgroups .............. 330

10. Group Schemes ........................................... 336

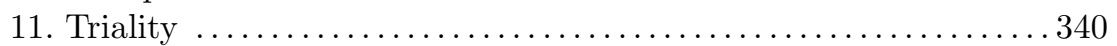

12. The Anisotropic Case ............................. 345

13. Appendix: Symmetric Spaces of Real Reductive Groups . . . . . . . 345

14. Appendix: The Symmetric Space of $G_{2}(\mathbb{R})$ and $G_{2}(\mathbb{C}) \ldots \ldots \ldots 352$

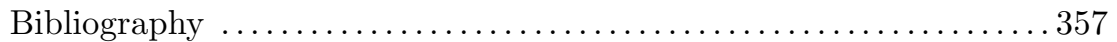

\section{Introduction}

The title of this paper is chosen as a tribute to the fundamental contributions of Bruhat and Tits to the structure theory of reductive groups over local fields through their series of papers [3], [4], [5], [7], [6], and is as far as we dare to venture with the French language. In [3], [4], Bruhat and Tits attach to any connected reductive group $G$ over a local field $k$ its building $\mathcal{B}(G)$, which is a polysimplicial complex equipped with an action of $G(k)$. To each point $x \in \mathcal{B}(G)$, they also attach a smooth connected affine group scheme $\underline{G}_{x}$ over the ring of integers $A$, with generic fiber $G$ and such that (at least when $G$ is simply-connected) $\underline{G}_{x}(A)$ is the stabilizer of $x$ in $G(k)$. The description of $\mathcal{B}(G)$ in [3], [4] is given in terms of the notion of valuations of root datum. However, in [5], [7], the building of a classical group $G$ is given a more concrete description in terms of the standard representation $V$ of $G: \mathcal{B}(G)$ is realized geometrically as a set of norms, or equivalently a set of graded lattice chains, on $V$ satisfying certain conditions, and the group schemes $\underline{G}_{x}$ are realized as stabilizers of these lattice chains in $V$. Using such a concrete description of $\mathcal{B}(G)$, one can give a lattice-theoretic description of the Moy-Prasad filtration on the parahoric subgroups of classical groups (cf. [20] and [19]). In view of such applications, it is useful to extend this concrete description of $\mathcal{B}(G)$ to the case when $G$ is an exceptional group, and the objective of the present paper is to carry out such a programme for the exceptional group of type $G_{2}$.

The reader familiar with Bruhat-Tits theory will be disappointed to learn that we will be working over a field which is complete with respect to a discrete valuation. Such a restriction would be considered a sin in [3], [4], but is already present to some extent in [5], [7]. Hence, throughout the paper, $A$ will denote a complete discrete valuation ring, with valuation map ord, field of fractions $k$,

TOME $131-2003-\mathrm{N}^{\mathrm{O}} 3$ 
uniformizer $\pi$, and perfect residue field $A / \pi$ of characteristic $p$. Let $G$ denote a simple algebraic group over $k$ of type $G_{2}$; we remind the reader that if the residue field $A / \pi$ has cohomological dimension $\leq 1$ (e.g. if $A / \pi$ is a finite field or is algebraically closed), then such a group is necessarily split [6]. Although all our main results are valid for an arbitrary form of $G_{2}$, we will assume that $G$ is the split form of $G_{2}$ in most part of the paper. The non-split case, which is very easy, is treated in $\S 12$.

The group $G$ can be constructed as the automorphism group of an octonion algebra $V$ over $k$, and thus has a natural 8-dimensional rational representation. We call $V$ the standard representation of $G$. Though this representation is not irreducible, it seems more natural to describe the building $\mathcal{B}(G)$ in terms of $V$, rather than, say, the 7-dimensional submodule of trace zero elements in $V$.

The octonion algebra $V$ possesses a natural quadratic form which is preserved by $G$. Hence the representation $V$ gives an embedding $\iota: G \hookrightarrow \operatorname{SO}(V)$. We show in $\S 4$ that this gives rise to a canonical embedding $\iota_{*}: \mathcal{B}(G) \hookrightarrow$ $\mathcal{B}(\mathrm{SO}(V))$. The building $\mathcal{B}(\mathrm{SO}(V))$ has been described explicitly in [7] as the set of maximinorante norms on $V$ (relative to the natural quadratic form on $V$ ). Our main results can now be summarized as follows.

(a) The determination of the image of $\iota_{*}$ (Thm. 7.2). The answer is most natural: $\mathcal{B}(G)$ is simply the set of maximinorante norms which are algebra norms for the octonion multiplication. This describes $\mathcal{B}(G)$ as a metric space.

(b) The description of the simplicial complex structure of $\mathcal{B}(G)$, in terms of certain orders in the octonion algebra (Thm. 9.5). Using these orders, we describe the parahoric subgroups of $G(k)$, as well as their associated smooth group schemes over $A$ (Thm. 10.1). We also describe the structure of apartments in $\mathcal{B}(G)$ (Prop. 8.1).

(c) There is a $S_{3}$-action on $\operatorname{Spin}(V)$ whose group of fixed points is $G$. This induces an action of $S_{3}$ on $\mathcal{B}(\operatorname{Spin}(V))$. We show that $\mathcal{B}(G)$ is precisely the set of points on $\mathcal{B}(\operatorname{Spin}(V))$ fixed under this action (Cor. 11.4).

The determination of the image of $\iota_{*}$ is an application of a general formalism described in $\S 3$ (Thm. 3.5). This formalism is quite useful for identifying the image of a descent map. In addition to (a) and (c), it can be applied to:

(d) The determination of the building of a classical group as a subset of the building of the ambient general linear group (Prop. 4.1). This reproves the results of [7] concerning the buildings of classical groups, at least when the residue characteristic $p$ is not 2 .

(e) An explicit description of the building of the split group $\operatorname{Spin}_{8}$ (Thm.11.3), together with the action of $S_{3}$; this will be needed in the study of the building of a general trialitarian $\operatorname{Spin}_{8}$.

When $p \neq 2,3$, the results (c) and (d) also follow from the general results of [13]. The proof here is valid also in residue characteristic 2 or 3 , and has the 
advantage/disadvantage of offering/requiring more information about the arithmetic and geometry underlying the groups involved. The formalism (Thm. 3.5) will also be useful in the study of the buildings of the other exceptional groups.

As is well-known, the reduced Bruhat-Tits building of $G(k)$ is the $p$-adic analogue of the symmetric space of a reductive real Lie group. In the Appendix $\S 13$, we introduce the notion of the extended symmetric space, which is the real analogue of the extended building and which has better functorial properties than the symmetric space. We also prove real analogues of (c) and (d), and more generally the analogue of the main theorem in [13]. Finally, in $\S 14$, we prove a real analogue of (a) (Thm. 7.2), which describes the symmetric space of $G_{2}(\mathbb{R})$ in terms of self-dual norms of an octonion algebra.

\section{Generalities on Norms}

In this section, let $V$ be a finite dimensional vector space over $k$. We shall recall some basic notions about norms on $V$. The material is largely taken from [5], [7], and we include it here for the convenience of the reader and for ease of reference.

A norm on $V$ is a function $\alpha: V \rightarrow \mathbb{R} \cup\{\infty\}$ satisfying:

$-\alpha(x+y) \geq \inf \{\alpha(x), \alpha(y)\}$, for all $x, y \in V$;

$-\alpha(\lambda x)=\operatorname{ord}(\lambda)+\alpha(x)$, for $\lambda \in k$ and $x \in V$;

$-\alpha(x)=\infty$ if and only if $x=0$.

A basis $\left\{x_{1}, \ldots, x_{n}\right\}$ of $V$ is called a splitting basis for $\alpha$ if

$$
\alpha\left(\sum_{i} \lambda_{i} x_{i}\right)=\inf _{i} \alpha\left(\lambda_{i} x_{i}\right)
$$

Since we are assuming that $k$ is complete with respect to a discrete valuation, every norm $\alpha$ possesses a splitting basis $[5,1.5]$. Moreover, if $\beta$ is another norm on $V$, there is a common splitting basis for $\alpha$ and $\beta$. For each $0 \leq t \leq 1$, there is a norm $\gamma_{t}$ which is characterized by the property that any common splitting basis $\left\{x_{1}, \ldots, x_{n}\right\}$ for $\alpha$ and $\beta$ is also a splitting basis for $\gamma_{t}$, and

$$
\gamma_{t}\left(x_{i}\right)=t \alpha\left(x_{i}\right)+(1-t) \beta\left(x_{i}\right), \quad i=1, \ldots, n .
$$

Another way of characterizing $\gamma_{t}$ is to say that it is the smallest norm satisfying

$$
\gamma_{t}(x) \geq t \alpha(x)+(1-t) \beta(x), \quad \text { for all } x \in V .
$$

We shall denote $\gamma_{t}$ by $t \alpha+(1-t) \beta$. This defines an affine structure on the set of norms on $V$.

The norm $\alpha$ determines a norm $\alpha^{*}$ on the dual space $V^{*}$, which is given by

$$
\alpha^{*}(\varphi)=\inf _{x \in V}(\operatorname{ord}(\varphi(x))-\alpha(x)) .
$$

TOME $131-2003-\mathrm{N}^{\mathrm{O}} 3$ 
More concretely, if $\left\{x_{1}, \ldots, x_{n}\right\}$ is a splitting basis for $\alpha$, then $\alpha^{*}$ is characterized by the requirement that it is split by the dual basis $\left\{x_{1}^{*}, \ldots, x_{n}^{*}\right\}$, and $\alpha^{*}\left(x_{i}^{*}\right)=-\alpha\left(x_{i}\right)$. Moreover, we have

$$
(t \alpha+(1-t) \beta)^{*}=t \alpha^{*}+(1-t) \beta^{*},
$$

for norms $\alpha$ and $\beta$ on $V$.

If $W$ is another finite-dimensional vector space over $k$, equipped with a norm $\beta$, then one can form a norm $\alpha \otimes \beta$ on $V \otimes W$, which is given as follows. Let $\left\{x_{1}, \ldots, x_{n}\right\}$ be a splitting basis for $\alpha$. Then any element of $V \otimes W$ can be written in the form $\sum_{i} x_{i} \otimes w_{i}$, and

$$
(\alpha \otimes \beta)\left(\sum_{i} x_{i} \otimes w_{i}\right)=\inf _{i}\left(\alpha\left(x_{i}\right)+\beta\left(w_{i}\right)\right) .
$$

In particular, since every non-zero vector is an element of a splitting basis for any norm, we have

$$
(\alpha \otimes \beta)(v \otimes w)=\alpha(v)+\beta(w), \quad \text { for } v, w \neq 0 .
$$

Now suppose that $V$ is equipped with a non-degenerate bilinear form $f$, and thus an isomorphism $V \rightarrow V^{*}$ given by: $x \mapsto f(x,-)$. Via this isomorphism, we can regard $\alpha^{*}$ as a norm on $V$, and we say that $\alpha$ is self-dual (with respect to the given bilinear form $f$ ) if $\alpha=\alpha^{*}$. By (1), one sees that the set of self-dual norms is a convex subset of the set of all norms, in the sense that $t \alpha+(1-t) \beta$ is self-dual if $\alpha$ and $\beta$ are.

There is another way of viewing the self-dual norms. Suppose that $(q, f)$ is a pair consisting of a non-degenerate quadratic form $q$ and the associated symmetric bilinear form $f$, so that

$$
f(x, y)=q(x+y)-q(x)-q(y) .
$$

Following [7], we say that a norm $\alpha$ minorizes $f$ if it satisfies

$$
\alpha(x)+\alpha(y) \leq \operatorname{ord}(f(x, y)), \quad \text { for all } x, y \in V .
$$

It was shown in [7, Prop. 2.5 (ii)] that $\alpha$ is self-dual with respect to $f$ if and only if it is a maximal element in the set of norms minorizing $f$. Similarly, say that $\alpha$ minorizes $(q, f)$ if it minorizes $f$, and satisfies

$$
\alpha(x) \leq \frac{1}{2} \cdot \operatorname{ord}(q(x)), \quad \text { for all } x \in V .
$$

If $\alpha$ is a maximal element in the set of norms minorizing $(q, f)$, then we say that $\alpha$ is maximinorante (suppressing the mention of $(q, f)$ ). Note that if the residue characteristic $p$ is not 2 , then $\alpha$ minorizes $f$ if and only if it minorizes $(q, f)$, and hence $\alpha$ is self-dual if and only if it is maximinorante. The situation is more complicated when $p=2$. For example, when $\operatorname{dim}(V)$ is odd, the form $f$ is degenerate if $\operatorname{char}(k)=2$, so that there is no notion of self-duality; even if $\operatorname{char}(k)=0$, it is possible to have a norm $\alpha$ which minorizes $f$ but not 
$(q, f)$, and hence a maximinorante norm need not be self-dual. However, as we shall see later, when $\operatorname{dim}(V)$ is even, a maximinorante norm is in fact always self-dual.

Assume further that $V$ is a $k$-algebra. We do not assume that the multiplication in $V$ is commutative or even associative. A norm $\alpha$ on $V$ is called an algebra norm if it satisfies

$$
\alpha(x \cdot y) \geq \alpha(x)+\alpha(y), \quad \text { for all } x, y \in V .
$$

In particular, if $V$ has a unit $e$, we have $\alpha(e) \leq 0$. We now make the following series of observations:

LEMMA 2.1. - Let $\left\{x_{1}, \ldots, x_{n}\right\}$ be a splitting basis for $\alpha$. For $\alpha$ to be an algebra norm, it is necessary and sufficient that

$$
\alpha\left(x_{i} \cdot x_{j}\right) \geq \alpha\left(x_{i}\right)+\alpha\left(x_{j}\right), \text { for all } i \text { and } j .
$$

Proof. - The necessity follows from definition. For the converse, let $x=$ $\sum_{i} \lambda_{i} x_{i}$ and $y=\sum_{j} \mu_{j} x_{j}$ be given. Then

$$
\begin{aligned}
\alpha(x \cdot y) & \geq \inf _{i, j}\left\{\alpha\left(\lambda_{i} x_{i} \cdot \mu_{j} x_{j}\right)\right\} \\
& \geq \inf _{i}\left\{\alpha\left(\lambda_{i} x_{i}\right)\right\}+\inf _{j}\left\{\alpha\left(\mu_{j} x_{j}\right)\right\} \\
& =\alpha(x)+\alpha(y) .
\end{aligned}
$$

This proves the lemma.

LEMMA 2.2. - The set of algebra norms is a convex subset of the set of all norms. In particular, the set of self-dual (respectively maximinorante) algebra norms is a convex subset of the set of self-dual (respectively maximinorante) norms.

Proof. - Suppose that $\alpha$ and $\beta$ are algebra norms, with common splitting basis $\left\{x_{1}, \ldots, x_{n}\right\}$. Let $\gamma_{t}$ denote $t \alpha+(1-t) \beta$. Then

$$
\begin{aligned}
\gamma_{t}\left(x_{i} \cdot x_{j}\right) & \geq t \alpha\left(x_{i} \cdot x_{j}\right)+(1-t) \beta\left(x_{i} \cdot x_{j}\right) \\
& \geq t \alpha\left(x_{i}\right)+(1-t) \beta\left(x_{i}\right)+t \alpha\left(x_{j}\right)+(1-t) \beta\left(x_{j}\right) \\
& =\gamma_{t}\left(x_{i}\right)+\gamma_{t}\left(x_{j}\right)
\end{aligned}
$$

Hence, $\gamma_{t}$ is also an algebra norm by Lemma 2.1 .

Now suppose that $E$ is a finite extension of $k$. The valuation ord on $k$ extends uniquely to a valuation, still denoted by ord, on $E$. Hence, we can form the norm $\alpha_{E}=\alpha \otimes$ ord on the $k$-vector space $V_{E}=V \otimes E$. Of course, $V_{E}$ has the natural structure of an $E$-algebra, and it is easy to see that $\alpha_{E}$ is in fact a norm of $E$-vector space. Further, we have:

LEMMA 2.3. - (i) $\left(\alpha_{E}\right)^{*}=\left(\alpha^{*}\right)_{E}$. In particular, $\alpha$ is self-dual if and only if $\alpha_{E}$ is self-dual.

TOME $131-2003-\mathrm{N}^{\mathrm{O}} 3$ 
(ii) If $\alpha$ is an algebra norm on $V$, then $\alpha_{E}$ is an algebra norm on the $E$ algebra $V_{E}$.

Proof. - Let $\left\{x_{1}, \ldots, x_{n}\right\}$ be a splitting basis for $\alpha$. Then it is also a basis for the $E$-vector space $V_{E}$, and is splitting for the norm $\alpha_{E}$. Moreover, if $\left\{x_{i}^{*}, \ldots, x_{n}^{*}\right\}$ is the dual basis, it is splitting for $\alpha^{*}$ and also for $\alpha_{E}^{*}$. Statement (i) then follows immediately from the definition of the dual norm, and statement (ii) follows by Lemma 2.1 .

Another way of viewing norms on $V$ is through the notion of graded lattice chains. Recall that a lattice chain in $V$ is a totally ordered (non-empty) set $L$. of lattices of $V$ (i.e. $A$-submodules $L$ of $V$ such that $L \otimes_{A} k=V$ ) which is stable under homotheties. Such a lattice chain can be represented as:

$$
L_{0} \supsetneq L_{1} \supsetneq \cdots \supsetneq L_{n-1} \supsetneq \pi L_{0},
$$

and the number $n$ is the rank of the lattice chain. A graded lattice chain is a pair $\left(L_{\bullet}, c\right)$ where $L_{\bullet}$ is a lattice chain and $c$ is a strictly decreasing map from $L$. to $\mathbb{R}$ such that

$$
c(\lambda \cdot L)=\operatorname{ord}(\lambda)+c(L) \quad \text { for } \lambda \in k \text { and } L \in L_{\bullet} .
$$

If the set of real numbers $\left\{c\left(L_{i}\right)-c\left(L_{i-1}\right): 0 \leq i \leq n-1\right\}$ generates a free abelian group of rank 1 , with positive generator $\delta$, then we say that the graded lattice chain $\left(L_{\bullet}, c\right)$ has period $\delta$.

A norm $\alpha$ on $V$ gives rise to a graded lattice chain $\left(L_{\alpha}, c_{\alpha}\right)$ as follows. The lattices in $L_{\alpha}$ are:

$$
L_{\alpha, r}=\{x \in V: \alpha(x) \geq r\} \quad \text { for } r \in \mathbb{R},
$$

and the map $c_{\alpha}$ is given by:

$$
c_{\alpha}\left(L_{\alpha, r}\right)=\inf _{x \in L_{\alpha, r}} \alpha(x) .
$$

Conversely, a graded lattice chain $\left(L_{\bullet}, c\right)$ gives rise to a norm $\alpha$ on $V$ as follows. For $x \in V$, let $L_{x}$ be the smallest member of $L$. containing $x$, and set $\alpha(x)=c\left(L_{x}\right)$. These two constructions are inverses of each other, and thus furnish a bijection between the set of norms on $V$ and the set of graded lattice chains. One can check that:

LEMma 2.4. - Suppose that $f$ is a non-degenerate bilinear form on $V$, and

$$
L_{0} \supsetneq L_{1} \supsetneq \cdots \supsetneq L_{n-1} \supsetneq \pi L_{0},
$$

with $c\left(L_{i}\right)=c_{i}$ is a graded lattice chain corresponding to a norm $\alpha$. Then the graded lattice chain $\left(L_{\bullet}^{*}, c^{*}\right)$ corresponding to the dual norm $\alpha^{*}$ (relative to $f$ ) is:

$$
\pi^{-1} L_{0}^{*} \supsetneq L_{n-1}^{*} \supsetneq \cdots \supsetneq L_{1}^{*} \supsetneq L_{0}^{*},
$$

with $c^{*}\left(L_{i}^{*}\right)=-c_{i-1}-\operatorname{ord}(\pi)$. 


\section{A General Formalism}

The goal of this section is to formulate a general approach which will allow one to identify the building of $G$ as a subset of the building of $H$ when $G$ is an algebraic subgroup of $H$. This formalism will be applied in later sections for the study of the buildings of exceptional groups. We begin by recalling some basic properties of buildings.

If $G$ is a connected reductive algebraic group over $k$, then its (extended) building $\mathcal{B}(G)$ is a set equipped with the following structures:

- $\mathcal{B}(G)$ is a complete metric space, with an affine structure;

- $\mathcal{B}(G)$ is the product of a polysimplicial complex and a real vector space;

- $G(k)$ acts isometrically on $\mathcal{B}(G)$ as simplicial automorphisms;

- $\mathcal{B}(G)$ has a collection of distinguished subsets, known as apartments, which are indexed by the maximal split tori of $G$.

If $T$ is a maximal split torus of $G$, then the corresponding apartment $\mathcal{A}(T)$ can be described as follows. Let $G_{1}$ be the derived group of $G$ and $Z(G)$ the connected center of $G$, so that there is a natural central isogeny $G_{1} \times Z(G) \rightarrow G$. Let $T_{1}$ and $T_{2}$ be the maximal split tori for $G_{1}$ and $Z(G)$ such that the image of $T_{1} \times T_{2}$ is $T$, so that we have:

$$
X_{\bullet}(T) \otimes \mathbb{Q}=\left(X_{\bullet}\left(T_{1}\right) \otimes \mathbb{Q}\right) \oplus\left(X_{\bullet}\left(T_{2}\right) \otimes \mathbb{Q}\right) .
$$

The reduced apartment $\mathcal{A}_{\text {red }}(T)$ corresponding to $T$ is a torsor for the real vector space

$$
\left(X_{\bullet}(T) \otimes \mathbb{R}\right) /\left(X_{\bullet}\left(T_{2}\right) \otimes \mathbb{R}\right),
$$

equipped with the structure of a simplicial complex. The apartment $\mathcal{A}(T)$ is the direct product

$$
\mathcal{A}(T)=\mathcal{A}_{\text {red }}(T) \times\left(X .\left(T_{2}\right) \otimes \mathbb{R}\right)
$$

of the reduced apartment with a real vector space, and is a torsor for $X_{\bullet}(T) \otimes \mathbb{R}$.

Note that the real vector space $X_{\bullet}(T) \otimes \mathbb{R}$ has a natural $\mathbb{Z}$-structure given by the lattice $X_{\bullet}(T)$. Hence, for a fixed point $x \in \mathcal{A}(T)$, it makes sense to speak of the $R$-valued points of $\mathcal{A}(T)$ relative to $x$, for any subring $\mathbb{Z} \subset R \subset \mathbb{R}$ : these are simply the points of $\mathcal{A}(T)$ which are the translates of $x$ by $X_{\bullet}(T) \otimes R$. Suppose that $x=\left(x_{1}, x_{2}\right) \in \mathcal{A}(T)$ is a point such that $x_{1}$ is a vertex in $\mathcal{A}_{\text {red }}(T)$, and $x_{2} \in X_{\bullet}\left(T_{2}\right) \otimes \mathbb{Q}$. We shall simply call the $\mathbb{Q}$-valued points of $\mathcal{A}(T)$ relative to $x$ the rational points of $\mathcal{A}(T)$. As suggested by the terminology, this notion is independent of the choice of the point $x$ of the above type. Moreover, for any $x \in \mathcal{B}(G)$, say that $x$ is a rational point if $x$ is a rational point of any apartment containing it; this notion is again well-defined. Once we have a natural rational structure on $\mathcal{A}(T)$, it makes sense to speak of rational affine functions $\varphi$ on $\mathcal{A}(T)$. A rational half-space $\mathcal{H}$ of $\mathcal{A}(T)$ is a subset of $\mathcal{A}(T)$ defined by the inequality $\varphi(x) \geq 0$ for some rational affine function $\varphi$. To be more precise, if $x_{0}$ is a rational point on $\mathcal{A}(T)$, the choice of a basis for $X_{\bullet}(T) \otimes \mathbb{Q}$

TOME $131-2003-\mathrm{N}^{\mathrm{O}} 3$ 
determines a coordinate system $\left(u_{i}\right)$ for $\mathcal{A}(T)$ with $x_{0}$ as origin. Then we want $\mathcal{H}$ to be the set of points $x$ in $\mathcal{A}(T)$ satisfying an inequality of the form

$$
\sum_{i} a_{i} u_{i}(x) \geq \lambda
$$

with $a_{i}$ and $\lambda$ in $\mathbb{Q}$. We say that a subset of $\mathcal{A}(T)$ is a rational polytope if it is the intersection of a finite number of rational half spaces. Note that in a rational polytope, the subset of rational points is dense.

Now let $E$ be a Galois extension of $k$. Then one can form the building $\mathcal{B}\left(G_{E}\right)$ of $G_{E}=G \times{ }_{k} E$. This is equipped with an action of $\operatorname{Gal}(E / k)$, and there is a natural embedding $j: \mathcal{B}(G) \hookrightarrow \mathcal{B}\left(G_{E}\right)$ which is $G(k)$-equivariant and whose image is contained in $\mathcal{B}\left(G_{E}\right) \operatorname{Gal}(E / k)$.

Proposition 3.1. - If $E / k$ is tamely ramified, then $\mathcal{B}(G)=\mathcal{B}\left(G_{E}\right)^{\operatorname{Gal}(E / k)}$.

Proof. - For the unramified case, this is proven in [3], and the general case is a well-known but unpublished result of G. Rousseau; recently, however, a simple proof has been provided by G. Prasad. We refer the reader to [12].

We are now ready to formulate the main result of this section.

Definition 3.2. - A descent datum $\iota_{*}$ consists of:

- an embedding $\iota: G \hookrightarrow H$ of connected reductive algebraic groups over $k$;

- an embedding $\iota_{*}: \mathcal{B}(G) \hookrightarrow \mathcal{B}(H)$ which is isometric, $G(k)$-equivariant and toral in the sense of [11].

This notion of descent data differs from that used in [3], where the definition is given in terms of valuations of root datum. The equivalence of the two definitions is verified in [18]. Following [18], we have:

Definition 3.3. - A strong descent datum $\left(\iota_{E *}\right)$ consists of:

- an embedding $\iota: G \hookrightarrow H$ of connected reductive algebraic groups over $k$;

- for each finite Galois extension $E$ of $k$, an embedding

$$
\iota_{E *}: \mathcal{B}\left(G_{E}\right) \longleftrightarrow \mathcal{B}\left(H_{E}\right)
$$

which is isometric, $G(E)$-equivariant, $\operatorname{Gal}(E / k)$-equivariant and toral in the sense of [11];

- for $E \subset F$ any finite Galois extensions of $k$, the diagram

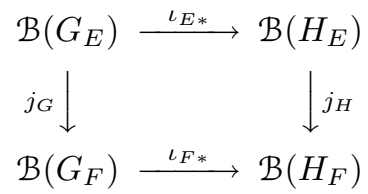

is commutative.

BULLETIN DE LA SOCIÉtÉ MATHÉMATIQUE DE FRANCE 
REMARK 3.4. - In [3], [4], the functoriality of the formation of buildings is largely undiscussed. This question is resolved in a recent paper of Landvogt [11], where he shows that given any $\iota: G \hookrightarrow H$ and any Galois extension $E$, a map $\iota_{E *}$ exists. In particular, the embedding $\iota_{E}: G_{E} \hookrightarrow H_{E}$ can be completed to a descent datum. What is not apparent from [11] is that one can find a compatible system of such embeddings $\iota_{E *}$ as required by the second and third conditions above, i.e. that $\iota$ can be completed to a strong descent datum. This turns out to be true fairly generally, for example if $G$ and $H$ are split by tamely ramified extensions. For more details, we refer the reader to [18].

Now suppose we are given a strong descent datum $\left\{\iota_{E *}\right\}$. Our goal is to determine the image of $\iota_{*}$. To this end, suppose we are given a subset $\mathcal{N}_{E} \subset$ $\mathcal{B}\left(H_{E}\right)$ for each $E$. Say that the collection $\left\{\mathcal{N}_{E}\right\}$ satisfies condition (BC) if

- $\mathcal{N}_{E}$ is convex and stable under the action of $G(E)$ for each $E$;

- for any $E \subset F$,

$$
j\left(\mathcal{N}_{E}\right) \subset \mathcal{N}_{F} .
$$

Let us write $\mathcal{N}$ in place of $\mathcal{N}_{k}$. Say that $\mathcal{N}$ satisfies condition (RAT) if $\mathcal{N} \cap \mathcal{A}$ is a rational polytope for any apartment $\mathcal{A}$ of $\mathcal{B}(H)$. We need to formulate one more property. For a rational point $x_{0} \in \mathcal{N}$, say that $x_{0}$ satisfies condition (TRANS) if the following holds:

- $x_{0}$ is the image of a rational point of $\mathcal{B}(G)$ under $\iota_{*}$;

- for each finite Galois extension $E$ of $k$, the set

$$
\mathcal{N}_{E}^{0}=\left\{x \in \mathcal{N}_{E}: x \text { is in the } H(E) \text {-orbit of } x_{0}\right\}
$$

is in the image of $\iota_{E *}$.

To verify condition (TRANS), it suffices to show that:

(i) $x_{0}$ is the image of a rational point under $\iota_{*}$;

(ii) $G(E)$ acts transitively on $\mathcal{N}_{E}^{0}$ for each $E$.

The main result of this section is:

THEOREM 3.5. - Given

- a strong descent datum $\left\{\iota_{E *}\right\}$;

- a collection $\left\{\mathcal{N}_{E} \subset \mathcal{B}\left(H_{E}\right)\right\}$ satisfying (BC) and (RAT);

- a rational point $x_{0} \in \mathcal{N}$ satisfying (TRANS).

Then the image of $\iota_{*}$ is $\mathcal{N}$.

Proof. - By definition, (TRANS) implies that $x_{0}$ is in the image of $\iota_{*}$. Since $\iota_{*}$ is $G(k)$-equivariant, and $\mathcal{N}$ is $G(k)$-invariant, the translates of $x_{0}$ by $G(k)$ lie in the image of $\iota_{*}$, as well as in $\mathcal{N}$. Now $\iota_{*}(\mathcal{B}(G))$ is a convex subset of $\mathcal{B}(H)$, and is in fact the convex hull in $\mathcal{B}(H)$ of the set of points $\left\{g \cdot x_{0}: g \in G(k)\right\}$. Since $\mathcal{N}$ is

TOME $131-2003-\mathrm{N}^{\mathrm{O}} 3$ 
convex, we have $\iota_{*}(\mathcal{B}(G)) \subset \mathcal{N}$, and it remains to prove the surjectivity of $\iota_{*}$ onto $\mathcal{N}$. Set

$$
\begin{aligned}
\mathcal{N}^{\prime}=\left\{x \in \mathcal{N}: x \text { is in the } H(E) \text {-orbit of } x_{0}\right. \text { for some } \\
\text { tamely ramified Galois extension } E / k\} .
\end{aligned}
$$

By (BC) and (TRANS), if $x \in \mathcal{N}^{\prime}$ is in the $H(E)$-orbit of $x_{0}$ with $E$ tamely ramified over $k, x$ lies in the image of $\iota_{E *}$. Hence

$$
x \in \iota_{E *}\left(\mathcal{B}\left(G_{E}\right)\right) \cap \mathcal{B}(H) .
$$

Since $\iota_{E *}$ is $\operatorname{Gal}(E / k)$-equivariant, Prop. 3.1 implies that $x \in \iota_{*}(\mathcal{B}(G))$. Hence we have shown that $\mathcal{N}^{\prime}$ is in the image of $\iota_{*}$. Since $\iota_{*}(\mathcal{B}(G))$ is a closed subset of $\mathcal{B}(H)$, it remains to prove that $\mathcal{N}^{\prime}$ is a dense subset of $\mathcal{N}$. More precisely, since

$$
\mathcal{N}=\bigcup_{\mathcal{A}: x_{0} \in \mathcal{A}} \mathcal{N} \cap \mathcal{A}
$$

it suffices to show that for each apartment $\mathcal{A}$ of $\mathcal{B}(H)$ containing $x_{0}, \mathcal{N}^{\prime} \cap \mathcal{A}$ is a dense subset of $\mathcal{N} \cap \mathcal{A}$. By (RAT), the rational points of $\mathcal{N} \cap \mathcal{A}$ are dense in $\mathcal{N} \cap \mathcal{A}$; hence it suffices to show that any rational point of $\mathcal{N} \cap \mathcal{A}$ can be approximated by points in $\mathcal{N}^{\prime} \cap \mathcal{A}$.

Let $T$ be the maximal split torus of $H$ corresponding to the apartment $\mathcal{A}$, and fixing the base point $x_{0}$ as the origin, we identify $\mathcal{A}$ with the real vector space $X_{\bullet}(T) \otimes \mathbb{R}$. Let

$$
L=\left\{t \cdot x_{0}: t \in T(k)\right\} .
$$

Then $L$ is a lattice in $\mathcal{A}$, with the property that $L \otimes \mathbb{Q}$ is precisely the set of rational points on $\mathcal{A}$, and for any extension $E / k$ with ramification index $e$,

$$
\frac{1}{e} \cdot L \subset\left\{x \in \mathcal{A}: x \text { is in the } H(E) \text {-orbit of } x_{0}\right\} .
$$

In particular, if $x \in \mathcal{N} \cap \mathcal{A}$ lies in $L \otimes \mathbb{Z}_{(p)}$, then $x \in \mathcal{N}^{\prime}$. Now let $x$ be any rational point in $\mathcal{N} \cap \mathcal{A}$, and consider the line $\{t x: 0 \leq t \leq 1\}$ joining $x_{0}$ to $x$. By convexity, this line lies in $\mathcal{N}$. Taking, for example, the sequence

$$
\frac{p^{n}}{p^{n}+1} x, \quad \text { for } n \text { sufficiently large, }
$$

we see that $x$ can be approximated by points in $\mathcal{N}^{\prime}$. The theorem is proved.

\section{The Buildings of Classical Groups}

In this section, we give two examples to illustrate the use of Thm. 3.5. The first example concerns buildings of classical groups. We will show that Thm. 3.5 gives quickly a new proof of one of the main results of [7] when the residue characteristic is $\neq 2$. Although our method can be applied to an arbitrary classical group (and even for some classical groups in residue characteristic 2, e.g. the symplectic groups) in a uniform way as in [7], the general setting 
requires too many notations. Hence we shall only concentrate on the case that will be used in this paper, namely the case of a special orthogonal group.

We begin by recalling some results from [5], [7]. Let $V$ be a finite dimensional vector space over $k$. In [5], the building of $\mathrm{GL}(V)$ is canonically identified with the set of norms on $V$. Moreover, the various structures on $\mathcal{B}(\mathrm{GL}(V))$ can all be described naturally in terms of norms. See also [8].

Proposition 4.1. - Let $(q, f)$ be a non-degenerate quadratic form on $V$, with associated symmetric bilinear form $f$, and let $H=\mathrm{SO}(q)$ be the associated special orthogonal group.

1) $\mathcal{B}(H)$ can be canonically identified with the set of maximinorante norms on $V$ (relative to $(q, f)$ ). In particular, when the residue characteristic $p$ is different from $2, \mathcal{B}(H)$ is the set of self-dual norms (relative to $f$ ).

2) Assume that $p \neq 2$. Let $g \mapsto g^{*}$ be the involution on $\operatorname{GL}(V)$ defined by

$$
f\left(g x, g^{*} y\right)=f(x, y), \quad \text { for all } x, y \in V .
$$

Then $H$ is the identity component of the algebraic subgroup fixed by the involution $*$, and the induced automorphism $*$ on $\mathcal{B}(\mathrm{GL}(V))$ is the map which sends a norm $\alpha$ to its dual $\alpha^{*}$. In particular, $\mathcal{B}(H)$ is the subset of $\mathcal{B}(\mathrm{GL}(V))$ fixed by $*$.

3) If $\operatorname{dim}(V)$ is even, a norm is maximinorante if and only if it is self-dual and minorizes $q$.

Proof. — The first two statements are special cases of results in [7]. It is easy to see that 1 ) and 2) are equivalent in case $p \neq 2$. We now give a new proof of 1) (and hence 2) using Thm. 3.5, under the assumption that $p \neq 2$ and $q$ is split.

Let $L_{0}$ be a self-dual lattice in $V$. Then $H(k) \cap \operatorname{Aut}\left(L_{0}\right)$ is a parahoric subgroup of $H(k)$ corresponding to a hyperspecial point $y_{0} \in \mathcal{B}(H)$. Similarly, $\operatorname{Aut}\left(L_{0}\right)$ determines a hyperspecial point $x_{0} \in \mathcal{B}(\mathrm{GL}(V))$. By a result of Bruhat and Tits [3, p. 203] recalled as Prop. 7.1 below, there is a strong descent datum $\left\{\iota_{E *}\right\}$ extending the embedding $\iota: H \hookrightarrow \operatorname{GL}(V)$ such that $\iota_{*}\left(y_{0}\right)=x_{0}$.

Let $\mathcal{N}_{E} \subset \mathcal{B}\left(\mathrm{GL}\left(V_{E}\right)\right)$ be the set of self-dual norms on $V_{E}$. It is easy to see that (BC) and (RAT) are satisfied. It remains to verify (TRANS). So let $\alpha$ be a self-dual norm on $V \otimes E$ such that $\alpha$ is $\operatorname{GL}\left(V_{E}\right)$-conjugate to $x_{0}$. Then $\alpha$ is completely determined by the lattice $L=\left\{v \in V_{E}: \alpha(v) \geq 0\right\}$, which is easily seen to be self-dual. Since $p \neq 2, L$ is $H(E)$-conjugate to $L_{0} \otimes \mathcal{O}_{E}$ and hence $\alpha$ is $H(E)$-conjugate to $x_{0}$. This proves (TRANS) and hence Thm. 3.5 implies that the image of $\iota_{*}$ is $\mathcal{N}$.

The identification of $\mathcal{B}(H)$ with $\mathcal{N}$ via $\iota_{*}$ is canonical in the sense that there is only one descent map from $\mathcal{B}(H)$ to $\mathcal{B}(\mathrm{GL}(V))$ whose image is fixed by *. This follows directly from [11, Cor. 2.7.4] since the centralizer of $H$ in GL( $V)$

TOME $131-2003-\mathrm{N}^{\mathrm{O}} 3$ 
is the center of $\mathrm{GL}(V)$. It also follows from a much more elementary result in $[18]$.

Statement 1) is now proven for split $q$.

Next, assuming $p \neq 2$, we will show that 2) can be reduced to the case that $q$ is split. It is well-known that there exists a splitting field $E$ for $H$ which is unramified or a ramified quadratic extension over an unramified extension. Since $p \neq 2, E / k$ is tamely ramified.

Since the action of $\phi=\{1, *\}$ on $\operatorname{GL}\left(V_{E}\right)$ is defined over $k$, it commutes with the action of $\Gamma=\operatorname{Gal}(E / k)$. Therefore, the action of $\phi$ on $\mathcal{B}(\mathrm{GL}(V \otimes E))$ also commutes with that of $\Gamma$. It follows that

$$
\mathcal{B}(H)=(\mathcal{B}(H \otimes E))^{\Gamma}=\left(\mathcal{B}\left(\operatorname{GL}\left(V_{E}\right)\right)^{\phi}\right)^{\Gamma}=\left(\mathcal{B}\left(\mathrm{GL}\left(V_{E}\right)\right)^{\Gamma}\right)^{\phi}=\mathcal{B}(\mathrm{GL}(V))^{\phi} .
$$

This proves 2). Here, we have identified $\mathcal{B}(H)$ with a subset of $\mathcal{B}(\operatorname{GL}(V))$ via $\iota_{*}$ and used the fact that the canonicity of $\iota_{*}$ implies that $\iota_{*}$ commutes with the action of $\Gamma$.

Now 1) and 2) have been proved in complete generality when $p \neq 2$.

For statement 3), if $\alpha$ is self-dual and minorizes $q$, it is clear that $\alpha$ must be maximinorante. To prove the converse, we first suppose that $(V, q)$ is split. Then by [7], one sees that the hyperspecial vertices of $\mathcal{B}(H)$ which correspond to self-dual lattices give rise to self-dual norms. Since the set of self-dual norms is convex and $H(k)$-invariant, and $\mathcal{B}(H)$ is the convex hull in $\mathcal{B}(\mathrm{GL}(V))$ of these hyperspecial vertices, we deduce that $\mathcal{B}(H)$ is contained in the set of selfdual norms. Hence, we have verified the statement in the split case. For the general case, let $E / k$ be a Galois extension over which $(V, q)$ is split. Then $\alpha_{E}$ lies on the building $\mathcal{B}\left(H_{E}\right)$ and hence is a maximinorante norm on $V_{E}$. By the split case, $\alpha_{E}$ is self-dual and minorizes $q$. By Lemma 2.3 (i), $\alpha$ is also self-dual and minorizes $q$.

We come now to the second illustration of Thm. 3.5. Suppose that $(V, q)$ is split, that $V$ is equipped with the structure of a $k$-algebra, and $G=\operatorname{Aut}(q, f, \cdot)$ is connected and reductive. We then have an embedding $\iota: G \hookrightarrow H$, where $H=\mathrm{SO}(q)$. Suppose that this can be completed to a strong descent datum $\left\{\iota_{E *}\right\}$. Then we want to identify the building $\mathcal{B}(G)$ as a subset of the set of maximinorante norms. To this end, let $\mathcal{N}_{E} \subset \mathcal{B}\left(H_{E}\right)$ be the subset of algebra norms, which is clearly $G(E)$-invariant. Lemmas 2.2 and 2.3 then imply that $\mathcal{N}_{E}$ satisfies (BC). It is also easy to check that $\mathcal{N}$ satisfies (RAT). Indeed, given an apartment $\mathcal{A}(T)$, a basis $\left\{x_{i}\right\}$ of $V$ consisting of weight vectors for $T$ is a splitting basis for every norm $\alpha$ corresponding to a point in $\mathcal{A}(T)$. Hence, $\alpha$ is completely determined by the collection $\left\{u_{i}(\alpha)=\alpha\left(x_{i}\right)\right\}$ of real numbers. A subset of this provides coordinates for $\mathcal{A}(T)$ for which the origin is a rational point. Now by Lemma 2.1, $\alpha$ lies in $\mathcal{N}$ if and only if $\alpha$ satisfies the system of inequalities:

$$
\alpha\left(x_{i} \cdot x_{j}\right) \geq \alpha\left(x_{i}\right)+\alpha\left(x_{j}\right) \text { for all } i, j .
$$

BULletin DE LA SOCiÉtÉ MATHÉMATIQUE DE FRANCE 
Writing $x_{i} \cdot x_{j}=\sum_{k} \lambda_{i j k} x_{k}$, the above condition is equivalent to

$$
\operatorname{ord}\left(\lambda_{i j k}\right)+u_{k}(\alpha) \geq u_{i}(\alpha)+u_{j}(\alpha) \text { for all } i, j, k \text {. }
$$

Hence, $\mathcal{N}$ satisfies (RAT), and as an application of Thm. 3.5, we have:

Proposition 4.2. - Let $\left\{\iota_{E *}\right\}$ be the given strong descent datum. Suppose further that

- the quadratic form $q$ is integral-valued on all orders of $V$;

- for each extension $E$ of $k, G(E)$ acts transitively on the orders $L_{E}$ of $V_{E}$ with $L_{E}$ a maximal lattice.

- there exists a hyperspecial point $\alpha_{0}$ which is the image of a rational point under $\iota_{*}$, and such that $L_{0}=\left\{x \in V: \alpha_{0}(x) \geq 0\right\}$ is an order which is a maximal lattice.

Then $\mathcal{B}(G)$ is the set of maximinorante norms which are algebra norms.

Proof. - It remains to verify that $\alpha_{0}$ satisfies (TRANS). The third assumption says that the lattice chain corresponding to $\alpha_{0}$ has rank 1 and its grading $c$ takes values in $\operatorname{ord}\left(k^{\times}\right)$with period $\operatorname{ord}(\pi)$. Hence, $\alpha_{0}$ is completely determined by $L_{0}$, with $c\left(L_{0}\right)=0$. Further, since $L_{0}$ is an order, $\alpha_{0}$ is an algebra norm, i.e. $\alpha_{0}$ lies in $\mathcal{N}$. For any $\alpha \in \mathcal{N}_{E}$, the lattice $L_{E}=\{x \in V: \alpha(x) \geq 0\}$ is an order in $V_{E}$. If $\alpha \in \mathcal{N}_{E}$ is in the $H(E)$-orbit of $\alpha_{0}$, then the graded lattice chain corresponding to $\alpha$ is in the $H(E)$-orbit of that for $\alpha_{0}$; in particular, it is also a rank 1 chain, with $L_{E}$ a maximal lattice of the quadratic space $(V, q)$. Since $L_{E}$ is conjugate to $L_{0}$ under $G(E)$ by the second assumption, $\alpha$ is conjugate to $\alpha_{0}$ under $G(E)$, and the proposition follows from Thm. 3.5.

\section{The Octonion Algebra}

We now begin our investigation of the building of $G_{2}$. In this section, we give the construction of the split octonion algebra over $\mathbb{Z}$, following Zorn. We refer the reader to [10] for general facts about octonion algebras alluded to below.

Let $W$ be a free $\mathbb{Z}$-module of rank 3 , and let $W^{*}=\operatorname{Hom}(W, \mathbb{Z})$. Fix an isomorphism of $\wedge^{3} W$ with $\mathbb{Z}$, which gives rise to isomorphisms

$$
\wedge^{2} W \longrightarrow W^{*}, \quad\left(\wedge^{2} W\right)^{*} \longrightarrow W,
$$

given by

$$
\left(w_{1} \wedge w_{2}, w\right) \longmapsto w_{1} \wedge w_{2} \wedge w \in \wedge^{3} W \cong \mathbb{Z} .
$$

Further, we have an isomorphism $\wedge^{2} W^{*} \rightarrow\left(\wedge^{2} W\right)^{*}$ which is given by:

$$
\left(\varphi_{1} \wedge \varphi_{2}, w_{1} \wedge w_{2}\right) \longmapsto \varphi_{1}\left(w_{1}\right) \varphi_{2}\left(w_{2}\right)-\varphi_{1}\left(w_{2}\right) \varphi_{2}\left(w_{1}\right) .
$$

This gives rise to an isomorphism

$$
\wedge^{2} W^{*} \longrightarrow W
$$

TOME $131-2003-\mathrm{N}^{\mathrm{O}} 3$ 
Let $\Lambda$ be the space of matrices of the form

$$
\left(\begin{array}{ll}
a & w \\
\varphi & b
\end{array}\right), \quad \text { with } a, b \in \mathbb{Z}, w \in W \text { and } \varphi \in W^{*} .
$$

This is a free $\mathbb{Z}$-module of rank 8 , and we define a multiplication on $\Lambda$ by:

$$
\left(\begin{array}{ll}
a & w \\
\varphi & b
\end{array}\right) \cdot\left(\begin{array}{ll}
a^{\prime} & w^{\prime} \\
\varphi^{\prime} & b^{\prime}
\end{array}\right)=\left(\begin{array}{cc}
a a^{\prime}+\varphi^{\prime}(w) & a w^{\prime}+b^{\prime} w-\varphi \wedge \varphi^{\prime} \\
a^{\prime} \varphi+b \varphi^{\prime}+w \wedge w^{\prime} & b b^{\prime}+\varphi\left(w^{\prime}\right)
\end{array}\right) .
$$

There is an anti-involution $x \mapsto \bar{x}$ on $\Lambda$ given by:

$$
\left(\begin{array}{ll}
a & w \\
\varphi & b
\end{array}\right) \longmapsto\left(\begin{array}{cc}
b & -w \\
-\varphi & a
\end{array}\right) .
$$

Set $\operatorname{Tr}(x)=x+\bar{x}$ and $q(x)=x \cdot \bar{x}$. Then $\operatorname{Tr}$ is a linear form and $q$ is a quadratic form on $\Lambda$, which are explicitly given by:

$$
\operatorname{Tr}:\left(\begin{array}{cc}
a & w \\
\varphi & b
\end{array}\right) \longmapsto a+b, \quad q:\left(\begin{array}{cc}
a & w \\
\varphi & b
\end{array}\right) \longmapsto a b-\varphi(w) .
$$

The quadratic form $q$ permits composition, i.e. satisfies

$$
q(x \cdot y)=q(x) \cdot q(y),
$$

so that the triple $(\Lambda, q, \cdot)$ is a composition algebra over $\mathbb{Z}$, with the identity matrix as the unit element $e$.

The symmetric bilinear form $f$ associated to $q$ takes the form

$$
f(x, y)=\operatorname{Tr}(x \cdot \bar{y})
$$

and $\Lambda$ is a self-dual lattice relative to $f$. Let $\left\{e_{1}, e_{2}, e_{3}\right\}$ be a basis of $W$ so that $e_{1} \wedge e_{2} \wedge e_{3} \in \wedge^{3} W$ is identified with $1 \in \mathbb{Z}$, and let $\left\{e_{-1}, e_{-2}, e_{-3}\right\}$ be the basis of $W^{*}$ determined by the requirement that $e_{-i}\left(e_{j}\right)=-\delta_{i j}$. Then $e_{1} \wedge e_{2}$ is identified with $-e_{-3}, e_{-1} \wedge e_{-2}$ is identified with $e_{3}$, and so on. Also let

$$
e_{-4}=\left(\begin{array}{ll}
1 & 0 \\
0 & 0
\end{array}\right), \quad e_{4}=\left(\begin{array}{ll}
0 & 0 \\
0 & 1
\end{array}\right) .
$$

Then, with respect to the basis $\left\{e_{i}\right\}$ of $\Lambda$, the quadratic form $q$ is given by

$$
q\left(\sum_{i} a_{i} e_{i}\right)=a_{1} a_{-1}+a_{2} a_{-2}+a_{3} a_{-3}+a_{4} a_{-4} .
$$

Hence, $\left\{e_{i}\right\}$ forms a Witt basis for the quadratic space $(\Lambda, q)$.

The characteristic polynomial of $x \in \Lambda$ is the polynomial

$$
q(\lambda \cdot e-x)=\lambda^{2}-f(x, e) \lambda+q(x),
$$

and it can be shown that $x$ satisfies its own characteristic polynomial. Though multiplication in $\Lambda$ is neither commutative nor associative, we have

$$
\operatorname{Tr}((x \cdot y) \cdot z)=\operatorname{Tr}(x \cdot(y \cdot z)),
$$

BULLETIN DE LA SOCIÉtÉ MATHÉMATIQUE DE FRANCE 
so that we can write $\operatorname{Tr}(x y z)$ unambiguously, and $(x, y, z)=\operatorname{Tr}(x y z)$ defines a trilinear form on $\Lambda$. This satisfies

$$
(x, y, z)=(z, x, y)
$$

and is alternating when restricted to the rank 7 submodule of trace zero elements in $\Lambda$. The multiplication in $\Lambda$ is completely determined by the quadratic form $q$ ( $c f$. [10, Thm. 33.19]), but it does not seem possible to write down a formula for the product in terms of $q$. However, the product can be recovered from $f$ and $(-,-,-)$ and the unit element $e$ by:

$$
f(x \cdot y, \bar{z})=(x, y, z),
$$

with $\bar{z}=f(z, e) e-z$.

As is well-known, the composition algebra $\Lambda$ is alternative. More precisely, let

$$
[x, y, z]=(x y) z-x(y z)
$$

be the associator of the elements $x, y, z$. Then we have

$$
[x, x, y]=[x, y, y]=0, \quad \text { for all } x, y \in \Lambda \text {. }
$$

This implies that the trilinear map $[-,-,-]$ is skew-symmetric:

$$
[x, y, z]=-[y, x, z]=-[x, z, y] \text {. }
$$

Sometimes it is convenient to work with a different multiplication on $\Lambda$, defined by

$$
x * y=\bar{x} \cdot \bar{y}
$$

With this new multiplication, one still has

$$
q(x * y)=q(x) \cdot q(y),
$$

so that $(\Lambda, q, *)$ is still a composition algebra, albeit without a unit. It is an example of a symmetric composition algebra in the sense of [10, §34], i.e. it satisfies:

$$
f(x * y, z)=f(x, y * z) .
$$

Moreover, we have

$$
x *(y * x)=q(x) y=(x * y) * x .
$$

All these identities can of course be stated in terms of the original multiplication, but they look neater when written in terms of $*$.

Let $B$ be any $\mathbb{Z}$-algebra. By the (split) octonion algebra over $B$, we mean the algebra $\Lambda \otimes B$ obtained from $\Lambda$, with all its structures described above, by base extension. In particular, we have the octonion algebra $V=\Lambda \otimes k$ over $k$. An element $x \in V$ is said to be integral if its characteristic polynomial has coefficients in $A$. An order in $V$ is an $A$-lattice which is a unital subring, and an order is said to be maximal if it is not contained in any larger one. Note that

TOME $131-2003-\mathrm{N}^{\mathrm{O}} 3$ 
any order is closed under conjugation and consists only of integral elements. The following result of van der Blij and Springer [2] will be crucial:

Proposition 5.1. - (i) Suppose that $L$ is a lattice in $V$ which is closed under multiplication, but which may not contain the unit element e. Then $L$ is a maximal order if and only if it is a maximal lattice for $(V, q)$ (or equivalently, it is self-dual with respect to $f$ ).

(ii) Any two maximal orders in $V$ are isomorphic.

(iii) If $L$ is a maximal order, any left (or right) ideal of $L$ is of the form $\pi^{n} L$ for $n \geq 0$.

For example, the lattice $R=\Lambda \otimes A$ is an order in $V$, and Prop. 5.1 (i) implies that it is a maximal order.

We conclude this section by describing another model for the octonion algebra $\Lambda$ over $\mathbb{Z}(c f$. $[10, \S 33 \mathrm{C}$, p. 458$])$. Let $M$ be the associative algebra of $2 \times 2$ matrices over $\mathbb{Z}$. It is a composition algebra of rank 4 over $\mathbb{Z}$, and thus possesses an anti-involution $x \mapsto \bar{x}$. The $\mathbb{Z}$-module $M \oplus M$, equipped with the multiplication

$$
(m, n) \cdot\left(m^{\prime}, n^{\prime}\right)=\left(m m^{\prime}-n^{\prime} \bar{n}, \bar{m} n^{\prime}+m^{\prime} n\right),
$$

and the anti-involution

$$
(m, n) \longmapsto(\bar{m},-n),
$$

is then a composition algebra of rank 8 over $\mathbb{Z}$. For $(i, j, k)=(1,2,3),(2,3,1)$, or $(3,1,2)$, the map

$$
\phi: \Lambda \longmapsto M \oplus M
$$

defined by:

$$
\sum_{s=1}^{8} a_{s} e_{s} \longmapsto\left(\left(\begin{array}{cc}
a_{-4} & -a_{i} \\
a_{-i} & a_{4}
\end{array}\right),\left(\begin{array}{cc}
a_{-j} & -a_{-k} \\
a_{k} & a_{j}
\end{array}\right)\right)
$$

defines an isomorphism of composition algebras over $\mathbb{Z}$, and we call $M \oplus M$ the Cayley-Dickson model for $\Lambda$. In particular, $(M \oplus M) \otimes A$ is isomorphic to the maximal order $R$ in $V=\Lambda \otimes k$.

The Cayley-Dickson construction above can in fact be generalized to produce other orders in $V$. Let $B$ be a composition algebra of rank 4 over $A$, with antiinvolution $x \mapsto \bar{x}$. In particular, $B \otimes k$ is a quaternion algebra over $k$ and $B$ is an order in $B \otimes k$. If $\lambda$ is an element of $A$, let $\operatorname{CD}(B, \lambda)=B \oplus B$ be the $A$-algebra with multiplication

$$
(m, n) \cdot\left(m^{\prime}, n^{\prime}\right)=\left(m m^{\prime}+\lambda n^{\prime} \bar{n}, \bar{m} n^{\prime}+m^{\prime} n\right)
$$

and anti-involution

$$
(m, n) \longmapsto(\bar{m},-n) .
$$

BULLETIN DE LA SOCIÉtÉ MATHÉMATIQUE DE FRANCE 
Then $\operatorname{CD}(B, \lambda)$ is a composition algebra of rank 8 over $A$, and is an order in the $k$-algebra $\operatorname{CD}(B, \lambda) \otimes k$. Note that the $k$-algebra $\operatorname{CD}(B, \lambda) \otimes k$ is isomorphic to $V$ if and only if $\lambda$ is in the image of the norm map $x \mapsto x \cdot \bar{x}$ of $B \otimes k$. Still more generally, one can let $J$ be a left ideal in $B$, and consider the $A$-module $\mathrm{CD}(B, J, \lambda)=B \oplus J$. Then the same formulas as above endow $\operatorname{CD}(B, J, \lambda)$ with the structure of a composition algebra of rank 8 over $A$. We will later return to these constructions in Prop. 9.8.

\section{The Automorphism Group}

Let $\underline{H}=\mathrm{SO}(\Lambda, q)$ denote the special orthogonal group of the quadratic space $(\Lambda, q)$. More precisely, $\underline{H}$ is the connected component of identity of the affine group scheme whose group of $B$-valued points consists of those $g \in \operatorname{Aut}(\Lambda \otimes B)$ satisfying

$$
q \circ g=q, \quad \operatorname{det}(g)=1 .
$$

In particular, $\underline{H}$ is also affine, and is a closed subgroup scheme of Aut $(\Lambda)$. It is well-known that each fiber of $\underline{H}$ is the split simple algebraic group of type $\mathrm{SO}_{8}$ over the relevant residue field. Let $\underline{G}$ be the automorphism group of the octonion algebra $(\Lambda, q, \cdot)$. Then it is known that each fiber of $\underline{G}$ is the split simple algebraic group of type $G_{2}$ over the relevant residue field. Since $\underline{G}$ is connected, it is a closed subgroup scheme of $\underline{H}$, and hence of $\operatorname{Aut}(\Lambda)$.

From the above, we see that both $\underline{H}$ and $\underline{G}$ have smooth fibers over each point of $\mathbb{Z}$. We claim that they are both flat over $\mathbb{Z}$, and hence are the Chevalley group schemes of the relevant type. For this, we use the following flatness criterion, which may be well-known, but for which we are unable to locate a precise reference.

Proposition 6.1. - Let $X$ be a noetherian scheme, and $S$ a connected regular noetherian scheme of dimension 1. Let $f: X \longrightarrow S$ be a morphism. Suppose that

(i) for each $s \in S$, the fiber $X_{s}$ is an irreducible and reduced variety;

(ii) $\operatorname{dim} X_{s}$ is independent of $s$;

(iii) $f$ has a section.

Then $f$ is flat.

Proof. — Without loss of generality, we assume that $S=\operatorname{Spec}(A)$, where $A$ is a discrete valuation ring with field of fractions $k$, and $X=\operatorname{Spec}(B)$ is an affine noetherian scheme. Let $X^{\prime}$ be the schematic closure in $X$ of its generic fiber $X_{k}$, so that we have a closed immersion $i: X^{\prime} \rightarrow X$. Since $S$ is regular of dimension $1, X^{\prime}$ is flat over $S$; ă indeed $X^{\prime}=\operatorname{Spec}\left(B / B_{\text {tor }}\right)$, where $B_{\text {tor }}$ is the ideal of elements in $B$ which are killed by a power of $\pi$ (the uniformizer of $A$ ). It remains to show that $i$ is an isomorphism. By (iii), $f$ has a section $S \rightarrow X$. Since $A$ is free of $\pi$-torsion, this section must factor through $X^{\prime}$, and thus the

TOME $131-2003-\mathrm{N}^{\mathrm{O}} 3$ 
special fiber $X_{s}^{\prime}$ is non-empty. By (ii), the flatness of $X^{\prime}$ and the fact that $i_{k}$ is an isomorphism on the generic fibers, we have:

$$
\operatorname{dim}\left(X_{s}\right)=\operatorname{dim}\left(X_{s}^{\prime}\right)=\operatorname{dim}\left(X_{k}\right) .
$$

By (i), we deduce that $i_{s}$ is an isomorphism. In other words, the ring homomorphism $B \rightarrow B / B_{\text {tor }}$ is an isomorphism on tensoring with $A / \pi$. This implies that $B_{\text {tor }}=\pi B_{\text {tor }}$, i.e. that $B_{\text {tor }}$ is $\pi$-divisible. Since $B$ is noetherian, this is not possible unless $B_{\text {tor }}$ is zero, i.e. unless $i$ is an isomorphism. The proposition is proved.

Corollary 6.2. - Both $\underline{H}$ and $\underline{G}$ are smooth reductive groups over $\mathbb{Z}$ in the sense of [9, exposé XIX], and are equal to the schematic closure of their generic fibers in $\operatorname{Aut}(\Lambda)$.

The corollary implies that we have a closed immersion $\underline{G} \rightarrow \underline{H}$ of Chevalley groups over $\mathbb{Z}$. For various computational purposes, it is desirable to have a more explicit description of this embedding. The Witt basis $\left\{e_{i}\right\}$ of $(\Lambda, q)$ defined in the last section gives rise to an épinglage for $\underline{H}$, as described in [7]. More precisely, let $\underline{T}$ be the subgroup scheme of $\underline{H}$ which preserves each line $\mathbb{Z} e_{i}$. Let $a_{i} \in X^{\bullet}(\underline{T})$ be the character of $\underline{T}$ which gives the action of $\underline{T}$ on $\mathbb{Z} e_{i}$. Then the fact that $\underline{T} \subset \underline{H}$ implies that $a_{i}+a_{-i}=0$. Hence, the set $\left\{a_{1}, a_{2}, a_{3}, a_{4}\right\}$ serves as coordinate functions on $\underline{T}$, and identifies $\underline{T}$ with $\mathbb{G}_{m}^{4}$. We shall represent a generic element of $\underline{T}$ as $t\left(a_{1}, a_{2}, a_{3}, a_{4}\right)$. In particular, $\underline{T}$ is a maximal split torus of $\underline{H}$, and the root subgroups of $\underline{H}$ relative to $\underline{T}$ are completely determined. To describe them, for any $i$ and $j$ in $\{ \pm 1, \pm 2, \pm 3, \pm 4\}$ with $i \neq \pm j$, and $\lambda \in \mathbb{G}_{a}$, let $u_{i, j}(\lambda)$ be the element of $\underline{H}$ given by:

$$
u_{i, j}(\lambda):\left\{\begin{array}{l}
e_{i} \mapsto e_{i}+\lambda e_{-j}, \\
e_{j} \mapsto e_{j}-\lambda e_{-i}, \\
e_{k} \mapsto e_{k}, \quad \text { if } k \neq i, j .
\end{array}\right.
$$

Then $u_{i, j}: \mathbb{G}_{a} \hookrightarrow \underline{H}$ is a closed immersion, whose image $U_{i, j}$ is the root subgroup of $\underline{H}$ relative to $\underline{T}$, corresponding to the root $a_{i}+a_{j}$. The collection $\left\{u_{i, j}: \mathbb{G}_{a} \rightarrow \underline{H}\right\}$ is a Chevalley system of épinglage.

We now consider the closed subgroup $\underline{G}$ of $\underline{H}$. One can describe a subgroup of $\underline{G}$ isomorphic to $\mathrm{SL}_{3}$ easily. Indeed, for $g \in \mathrm{SL}_{3}$, the map

$$
g:\left(\begin{array}{ll}
a & w \\
\varphi & b
\end{array}\right) \longmapsto\left(\begin{array}{cc}
a & g w \\
\varphi \circ g^{-1} & b
\end{array}\right)
$$

defines an element of $\underline{G}$, and thus defines a closed immersion $\mathrm{SL}_{3} \hookrightarrow \underline{G}$. The diagonal torus $\underline{S}$ of $\mathrm{SL}_{3}$ thus serves as a maximal split torus for $\underline{G}$, and one sees easily that $\underline{S}$ is in fact the intersection of $\underline{G}$ with $\underline{T}$. Indeed, the embedding 
$\underline{S} \hookrightarrow \underline{T}$ is given by:

$$
\left(\begin{array}{lll}
a & 0 & 0 \\
0 & b & 0 \\
0 & 0 & c
\end{array}\right) \longmapsto t(a, b, c, 1), \quad \text { with } a b c=1 .
$$

The root subgroups of $\mathrm{SL}_{3}$ relative to $\underline{S}$ are precisely the root subgroups of $\underline{G}$ corresponding to the 6 long roots. As subgroups of $\underline{H}$, we have, for example,

$$
\left(\begin{array}{ccc}
1 & \lambda & 0 \\
0 & 1 & 0 \\
0 & 0 & 1
\end{array}\right) \mapsto u_{2,-1}(\lambda),\left(\begin{array}{ccc}
1 & 0 & 0 \\
0 & 1 & \lambda \\
0 & 0 & 1
\end{array}\right) \mapsto u_{3,-2}(\lambda),\left(\begin{array}{ccc}
1 & 0 & \lambda \\
0 & 1 & 0 \\
0 & 0 & 1
\end{array}\right) \mapsto u_{3,-1}(\lambda)
$$

Any other root subgroup of $\underline{H}$ relative to $\underline{S}$ is isomorphic to $\mathbb{G}_{a}^{3}$, given, for example, by:

$$
\left\{\begin{array}{l}
U_{1,3} \times U_{4,-2} \times U_{-2,-4}, \\
U_{3,2} \times U_{4,-1} \times U_{-1,-4}, \\
U_{-2,-1} \times U_{-4,3} \times U_{3,4} .
\end{array}\right.
$$

The intersection of these with $\underline{G}$ is, up to signs, given by the diagonal embedding of $\mathbb{G}_{a}$. Thus for any root $\gamma$ of $\underline{G}$ relative to $\underline{S}$, we have described the corresponding root subgroup $U_{\gamma}$ and an isomorphism $u_{\gamma}: \mathbb{G}_{a} \rightarrow U_{\gamma}$. The collection $\left\{u_{\gamma}: \mathbb{G}_{a} \rightarrow \underline{G}\right\}$ is a Chevalley system of épinglage. One can choose a system of simple roots $\{a, b\}$ for $\underline{G}$ relative to $\underline{S}$ by insisting that

$$
U_{b}=U_{2,-1}, \quad U_{a}=\underline{G} \cap\left(U_{1,3} \times U_{4,-2} \times U_{-2,-4}\right) .
$$

The following lemma is now straightforward:

LEMMA 6.3. - The image of the natural map

$$
X_{\bullet}(\underline{S}) \otimes \mathbb{R} \longleftrightarrow X .(\underline{T}) \otimes \mathbb{R}
$$

is given by the equations:

$$
a_{1}+a_{2}+a_{3}=0, \quad a_{4}=0 .
$$

Further, when restricted to $\underline{S}$, we have:

$$
\begin{array}{ll}
a_{1}=-a-b, & a_{2}=-a, \\
a_{3}=2 a+b, & a_{4}=0 .
\end{array}
$$

Using the Cayley-Dickson model $M \oplus M$ for $\Lambda$, we can describe the root subgroups of $\underline{G}$ corresponding to the short roots. Indeed, there is an embedding $j$ of $\mathrm{SL}_{2}$ into the automorphism group of $M \oplus M$ given by:

$$
j(g):(m, n) \longmapsto\left(g m g^{-1}, g n\right) .
$$

From this, we obtain three embeddings $\mathrm{SL}_{2} \hookrightarrow \underline{G}$, given by:

$$
g \longmapsto \phi_{i}^{-1} \circ j(g) \circ \phi_{i}
$$

TOME $131-2003-\mathrm{N}^{\mathrm{O}} 3$ 
where $\phi_{i}: \Lambda \cong M \oplus M$ is defined in (4) and (5). In particular, we have three embeddings of the root subgroups of $\mathrm{SL}_{2}$ relative to the diagonal torus, and these are the root subgroups of $\underline{G}$ relative to $\underline{S}$ corresponding to the short roots.

\section{The Building $\mathcal{B}(G)$}

Henceforth, let

$$
G=\underline{G} \times k, \quad H=\underline{H} \times k .
$$

In this section, we give a concrete description of the building $\mathcal{B}(G)$ using norms on the standard representation $V$ of $G$.

We have constructed in the previous section an embedding $\underline{G} \hookrightarrow \underline{H}$ of Chevalley groups, as well as maximal split tori $\underline{S} \subset \underline{G}$ and $\underline{T} \subset \underline{H}$ satisfying $\underline{S}=\underline{T} \cap \underline{G}$. The system $\varphi_{H}=\left(\varphi_{i, j}\right)$ of maps

$$
\varphi_{i, j}: U_{i, j}(k) \longrightarrow \mathbb{R}
$$

defined by

$$
u_{i, j}(\lambda) \longmapsto \operatorname{ord}(\lambda)
$$

is a valuation of the root datum $\left(T(k), U_{i, j}(k)\right)$, and defines a hyperspecial point $x_{0}$ on the apartment $\mathcal{A}(T)$ of $\mathcal{B}(H)$ determined by $T$. The stabilizer of $x_{0}$ in $H(k)$ is the maximal bounded subgroup $\underline{H}(A)$. Similarly, the system $\varphi_{G}=\left(\varphi_{\gamma}\right)$ of maps

$$
\varphi_{\gamma}: U_{\gamma} \longrightarrow \mathbb{R}
$$

defined by

$$
u_{\gamma}(\lambda) \longmapsto \operatorname{ord}(\lambda)
$$

is a valuation of the root datum $\left(S(k), U_{\gamma}(k)\right)$, and defines a hyperspecial point $x_{0}^{\prime}$ on the apartment $\mathcal{A}(S)$ of $\mathcal{B}(G)$ determined by $S$, whose stabilizer in $G(k)$ is the maximal bounded subgroup $\underline{G}(A)$.

Proposition 7.1. - The valuation $\varphi_{H}$ descends to the valuation $\varphi_{G}$. There exists a strong descent datum $\left\{\iota_{E *}: \mathcal{B}\left(G_{E}\right) \rightarrow \mathcal{B}\left(H_{E}\right)\right\}$, which is uniquely determined by the property that $\iota_{*}\left(x_{0}^{\prime}\right)=x_{0}$. Further, the map $\iota_{*} \operatorname{sends} \mathcal{A}(S)$ into $\mathcal{A}(T)$.

Proof. - It suffices to apply the general result on descent mappings for Chevalley groups in [3, p. 203]. The proof given there for this general result involves passing to a field extension of $k$ for which the image of the valuation map ord is $\mathbb{R}$. If one is uncomfortable with this procedure, one can prove the result for the case at hand, using the explicit information given in the last section. Indeed, from (6), it is easy to verify that $\varphi_{H}$ descends to $\varphi_{G}$; one can then apply [3, Prop. 9.1.17]. 
If we identify $\mathcal{A}(S)$ and $\mathcal{A}(T)$ with $X_{\bullet}(S) \otimes \mathbb{R}$ and $X_{\bullet}(T) \otimes \mathbb{R}$ by choosing $x_{0}^{\prime}$ and $x_{0}$ as origins, the map $\mathcal{A}(S) \rightarrow \mathcal{A}(T)$ is the natural one $X_{\bullet}(S) \otimes \mathbb{R} \rightarrow X_{\bullet}(T) \otimes \mathbb{R}$, which was described in coordinates in Lemma 6.3. Moreover, the functions $\left\{a_{1}, a_{2}, a_{3}, a_{4}\right\}$ serve as coordinates for $\mathcal{A}(T)$. The norm corresponding to $p \in \mathcal{A}(T)$ is given by $[7, \S 2.9]$ :

$$
\alpha_{p}\left(\sum \lambda_{i} e_{i}\right)=\inf _{i}\left\{\operatorname{ord}\left(\lambda_{i}\right)-a_{i}(p)\right\}
$$

We would now like to apply Prop. 4.2 to the strong descent datum $\left\{\iota_{E *}\right\}$ provided by Prop. 7.1. Hence, we let $\mathcal{N}_{E}$ be the set of maximinorante norms on $V$ which are norms of the octonion algebra, and it remains to verify that the hypotheses of Prop. 4.2 are satisfied. The fact that $q$ is integral on any order of $V$ follows from the fact that any order consists of integral elements. Let $\alpha_{0}$ be the maximinorante norm on $V$ corresponding to the hyperspecial point $x_{0}$. Then from $(7)$, one sees easily that $\left\{x \in V: \alpha_{0}(x) \geq 0\right\}=R$, which is an order, as well as a maximal lattice; hence the third condition of Prop. 4.2 holds. Finally, the second condition is a consequence of Prop. 5.1 (i) and (ii). We have verified all the hypotheses of Prop. 4.2, and thus proven:

THEOREM 7.2. - The map $\iota_{*}$ establishes a bijection between the building $\mathcal{B}(G)$ of $G$ and the set $\mathcal{N}$ of maximinorante norms for $(V, q)$ which are norms of the octonion algebra. In this bijection, the hyperspecial points of $\mathcal{B}(G)$ correspond to those algebra norms $\alpha$ which take values in $\operatorname{ord}(k)$, which in turn corresponds to maximal orders in $V$.

REMARK 7.3. - The map $\iota_{*}$ is canonical in the sense that it is the unique descent mapping from $\mathcal{B}(G)$ to $\mathcal{B}(\mathrm{SO}(V, q))$. Again, this follows either from [11, Cor. 2.7.4], or from an elementary result in [18].

We can also consider the descent datum consisting of the compositions $j$ : $G \hookrightarrow \mathrm{SO}(V, q) \hookrightarrow \mathrm{GL}(V)$ and

$$
j_{*}: \mathcal{B}(G) \longleftrightarrow \mathcal{B}(\mathrm{SO}(V, q)) \longleftrightarrow \mathcal{B}(\mathrm{GL}(V)) .
$$

By another application of Thm. 3.5 and Prop. 5.1, we can easily show:

THEOREM 7.4. - The image of $j_{*}$ is the set of self-dual norms for $f$ which are also norms of the octonion algebra.

As a consequence, we see that an algebra norm which is self-dual for $f$ is maximinorante for $q$. This fact can also be checked directly. We omit the details. Instead, let us record the following corollary:

Corollary 7.5. - Let $\alpha$ be a self-dual algebra norm. Then $\alpha(e)=0$ and $\alpha(\bar{x})=\alpha(x)$ for any $x \in V$.

TOME $131-2003-\mathrm{N}^{\mathrm{O}} 3$ 
Proof. - The fact that $\alpha$ is an algebra norm implies that $\alpha(e) \leq 0$. On the other hand, the self-dual algebra norm $\alpha_{0}$ corresponding to the hyperspecial point $x_{0}$ satisfies $\alpha_{0}(e) \geq 0$. The same is thus true for all self-dual algebra norms corresponding to hyperspecial vertices. Since $\mathcal{B}(G)$ is the convex hull in $\mathcal{B}(\mathrm{SO}(V, q))$ of all these hyperspecial vertices, we see that $\alpha(e) \geq 0$ for any $\alpha \in \mathcal{B}(G)$. The first assertion is proved.

Since $\bar{x}=f(x, e) e-x$, we see that $\alpha(\bar{x}) \geq \alpha(x)$ for any $\alpha \in \mathcal{B}(G)$. The second assertion thus follows.

It seems that one should be able to prove this corollary directly, without using the theory of buildings as we have done here.

\section{The Structure of Apartments}

We would now like to describe the various structures on $\mathcal{B}(G)$ directly in terms of $\mathcal{N}$. The affine structure of $\mathcal{B}(G)$ corresponds to the natural one on $\mathcal{N}$ discussed in Section 2, and the $G(k)$-action is clear. This section is devoted to a description of the apartments of $\mathcal{B}(G)$.

The apartments of $\mathcal{B}(G)$ are parametrized by the maximal split tori of $G$. A maximal split torus $T$ of $G$ is contained in a subgroup of $G$ which is isomorphic to $\mathrm{SL}_{3}$, and which is the pointwise stabilizer of a sub-algebra $k \times k$ of $V$. Such a sub-algebra has a unique orthogonal system of primitive idempotents $\left\{u_{4}, u_{-4}\right\}$, i.e. a pair of non-trivial idempotents $\left\{u_{4}, u_{-4}\right\}$ with $u_{4}+u_{-4}=e$ and $u_{4} \cdot u_{-4}=u_{-4} \cdot u_{4}=0$. These form a basis of the zero weight spaces for the action of $T$ on $V$. Conversely, suppose we are given an orthogonal system of primitive idempotents $\left\{u_{4}, u_{-4}\right\}$. Then they generate a sub-algebra of $V$ isomorphic to $k \times k$. By [10, Cor. 33.21], all such sub-algebras are conjugate under $G(k)$ to the one spanned by $\left\{e_{4}, e_{-4}\right\}$, i.e. the diagonal matrices in the Zorn's model for $V$. Hence the stabilizer in $G$ of $u_{4}$ is isomorphic to $\mathrm{SL}_{3}$. Further, for $i= \pm 4$,

$$
V_{i}=\left\{x \in V: u_{i} \cdot x=0 \text { and } x \cdot u_{i}=x\right\}
$$

is 3-dimensional, and preserved by $\mathrm{SL}_{3}$. The representations of $\mathrm{SL}_{3}$ on $V_{4}$ and $V_{-4}$ are isomorphic to the standard representation and its dual. Indeed, the symmetric bilinear form $f$ provides a non-degenerate $\mathrm{SL}_{3}$-invariant pairing between $V_{4}$ and $V_{-4}$. Now to give a maximal torus for $\mathrm{SL}_{3}$, it is necessary and sufficient to give a decomposition of $V_{4}$ into a direct sum of one-dimensional spaces $k u_{1} \oplus k u_{2} \oplus k u_{3}$. Note that by duality, this induces a decomposition $V_{-4}=k u_{-1} \oplus k u_{-2} \oplus k u_{-3}$, where $f\left(u_{i}, u_{-j}\right)=-\delta_{i j}$. Concluding, we have shown:

Proposition 8.1. - To give an apartment of $\mathcal{B}(G)$, it is necessary and sufficient to give the following data: 
- an orthogonal system of primitive idempotents $\left\{u_{4}, u_{-4}\right\}$ of $V$;

- a decomposition of $V_{4}=\left\{x \in V: u_{4} \cdot x=0\right.$ and $\left.x \cdot u_{4}=x\right\}$ into a direct sum of one-dimensional spaces $k u_{1} \oplus k u_{2} \oplus k u_{3}$.

Given such a data, the corresponding apartment is the subset of $\mathcal{N}$ consisting of those algebra norms which are split by the basis $\left\{u_{i}: i= \pm 1, \pm 2, \pm 3, \pm 4\right\}$ of $V$.

\section{Simplicial Structure and Parahoric Subgroups}

In this section, we describe the simplicial structure of $\mathcal{B}(G)$. More precisely, we view the vertices of the simplicial complex $\mathcal{B}(G)$ in terms of graded lattice chains in $V$, and describe the incidence relation. Concurrently, we realize the parahoric subgroups of $G(k)$ as stabilizers of certain orders of $V$.

Henceforth, we assume $\operatorname{ord}(\pi)=1$. Let us identify the apartments $\mathcal{A}(S)$ and $\mathcal{A}(T)$ with the real vector spaces $X_{\bullet}(S) \otimes \mathbb{R}$ and $X_{\bullet}(T) \otimes \mathbb{R}$ by choosing $x_{0}^{\prime}$ and $x_{0}$ as origins, and let us regard $\mathcal{A}(S)$ as a subset of $\mathcal{A}(T)$ using $\iota_{*}$. Having fixed the origin, the simple roots $a$ and $b$ become affine functions on $\mathcal{A}(S)$, and serve as coordinates for $\mathcal{A}(S)$. Let $c=3 a+2 b$ be the highest root, and consider the closed chamber in $\mathcal{A}(S)$ defined by:

$$
C_{G}=\{p \in \mathcal{A}(S): a(p) \geq 0, b(p) \geq 0,1 \geq c(p)\} .
$$

Then $C_{G}$ looks like the shaded area in the following figure:

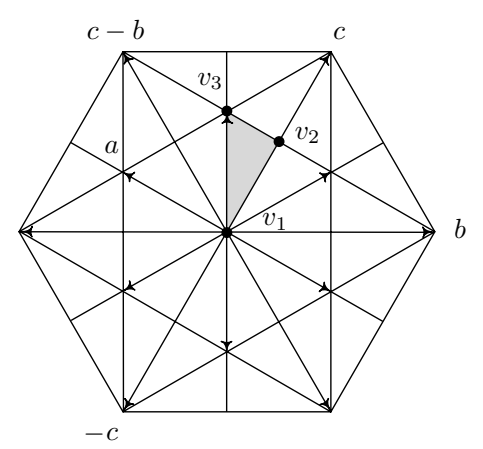

The vertices have coordinates $(a, b)$ given by:

$$
v_{1}=(0,0), \quad v_{2}=\left(0, \frac{1}{2}\right), \quad v_{3}=\left(\frac{1}{3}, 0\right) .
$$

In particular, the vertex $v_{1}$ is the hyperspecial point $x_{0}^{\prime}$, and we say that a vertex of $\mathcal{B}(G)$ is of type $i$ if it is conjugate under $G(k)$ to $v_{i}$.

TOME $131-2003-\mathrm{N}^{\mathrm{O}} 3$ 
As we have seen in Section 6 , the functions $\left\{a_{1}, a_{2}, a_{3}, a_{4}\right\}$ serve as coordinates on $\mathcal{A}(T)$. We fix the simple roots of $H$ relative to $T$ to be:

$$
\begin{array}{ll}
r_{1}=a_{3}+a_{1}, & r_{2}=-a_{1}+a_{2}, \\
r_{3}=-a_{2}+a_{4}, & r_{4}=-a_{2}-a_{4} .
\end{array}
$$

Then $\left\{r_{1}, r_{2}, r_{3}, r_{4}\right\}$ also serve as coordinates on $\mathcal{A}(T)$. The highest root is $r_{0}=r_{1}+2 r_{2}+r_{3}+r_{4}$, and the inequalities:

$$
1 \geq r_{0}, \quad r_{i} \geq 0, \quad i=1,2,3,4,
$$

define a closed chamber $C_{H}$ of $\mathcal{B}(H)$. Using Lemma 6.3 , one sees easily that when regarded as points on $\mathcal{A}(T)$, the vertices $v_{1}, v_{2}, v_{3}$ have $\left(r_{i}\right)$-coordinates given by:

$$
v_{1}=(0,0,0,0), \quad v_{2}=\left(0, \frac{1}{2}, 0,0\right), \quad v_{3}=\left(\frac{1}{3}, 0, \frac{1}{3}, \frac{1}{3}\right) .
$$

In particular, $v_{1}$ and $v_{2}$ are vertices of the chamber $C_{H}$, whereas $v_{3}$ is the barycenter of the triangle formed by the other 3 vertices of $C_{H}$. Moreover, it is easy to check that $C_{H} \cap \mathcal{A}(S)=C_{G}$.

We have seen in Thm. 7.2 that the hyperspecial vertices of $\mathcal{B}(G)$ are identified by $\iota_{*}$ with those maximinorante norms of algebra which take values in $\operatorname{ord}(k)$, which in turn correspond to maximal orders of $V$. More precisely, the lattice chains corresponding to such norms are of the form

$$
\cdots \subsetneq \pi L \subsetneq L \subsetneq \cdots
$$

with $L$ a maximal order. We denote the set of maximal orders by $\mathcal{V}_{1}$.

Now consider the vertices of type 2 . The vertex $v_{2}$ determines a graded lattice chain $\left(L_{\bullet}, c\right)$ in $V$ of the type:

$$
\cdots \subsetneq \pi L \subsetneq \pi L^{*} \subsetneq L \subsetneq \cdots
$$

with $c(L)=0$, and $c\left(L^{*}\right)=-\frac{1}{2}$. Using $(7)$, one sees that

$$
L=\left\langle e_{1}, e_{2}, \pi e_{3}, e_{4}, \pi e_{-1}, e_{-2}, e_{-3}, e_{-4}\right\rangle .
$$

Moreover, since $v_{2}$ corresponds to a norm of algebra, we have:

$$
\left\{\begin{array}{l}
L \text { is an order, and } \\
\pi L^{*} \text { is an ideal of } L \text { satisfying }\left(\pi L^{*}\right)^{2} \subset \pi L .
\end{array}\right.
$$

Let $\mathcal{V}_{2}$ be the set of orders $L$ satisfying

$$
L \subsetneq L^{*} \subsetneq \pi^{-1} L, \quad L^{* 2} \subset \pi^{-1} L .
$$

Here, $L^{* 2}$ is the $A$-lattice spanned by all elements $x \cdot y$, with $x, y \in L^{*}$. Since the set $\mathcal{V}_{2}$ is clearly stabilized by the action of $G(k)$, we have shown that there is an injection from the set of vertices of type 2 into $\mathcal{V}_{2}$. Now we have:

Lemma 9.1. - The set of vertices of type 2 is in bijection with $\mathcal{V}_{2}$. 
Proof. - It remains to show that the injection above is surjective. Suppose we are given a $L$ in $V_{2}$. Let $\left(L_{\bullet}, c\right)$ be the lattice chain

$$
\cdots \subsetneq \pi L \subsetneq \pi L^{*} \subsetneq L \subsetneq \cdots
$$

with grading $c(L)=0$ and $c\left(L^{*}\right)=-\frac{1}{2}$. This gives rise to a norm $\alpha$ on $V$, which is easily seen to be self-dual. On the other hand, (3) implies that

$$
f(x \cdot y, z)=f(y, \bar{x} \cdot z)=f(x, z \cdot \bar{y}) .
$$

Now suppose $x \in L$ and $y \in L^{*}$. Then for any $z \in L$, we have:

$$
f(x \cdot y, z)=f(y, \bar{x} \cdot z) \in A,
$$

so that $L \cdot L^{*} \subset L^{*}$, and a similar argument gives $L^{*} \cdot L \subset L^{*}$. From this, one sees that $\pi L^{*}$ is an ideal of $L$, and thus $\alpha$ is an algebra norm. By Thm. 7.4, $\alpha$ is a point on $\mathcal{B}(G)$.

It remains to show that $\alpha$ is a vertex of type 2 . Conjugating by an element of $G(k)$, we may assume $\alpha$ is a point in the closed chamber $C_{G}$ described above, and we need to show that $\alpha$ is equal to $v_{2}$. Now the image of the norm $\alpha$ is $\frac{1}{2} \mathbb{Z}$, which implies by $(7)$ and Lemma 6.3 that as a point on $\mathcal{A}(S)$, its coordinates $a$ and $b$ lie in $\frac{1}{2} \mathbb{Z}$, but not both in $\mathbb{Z}$. Since $a$ and $b$ must also satisfy (8), it is easy to see that the only possibility is

$$
a=0, \quad b=\frac{1}{2} .
$$

In other words, $\alpha$ is equal to $v_{2}$, and the set of vertices of type 2 are in bijection with $\mathcal{V}_{2}$.

Corollary 9.2. - If $L \in \mathcal{V}_{2}$, then length $_{A}\left(L^{*} / L\right)=4$. Indeed, $L / \pi L^{*}$ is isomorphic to the algebra of $2 \times 2$ matrices over $A / \pi$.

We now come to vertices of type 3 . The vertex $v_{3}$ gives rise to the graded lattice chain $\left(L_{\bullet}, c\right)$ :

$$
\cdots \pi L \subsetneq \pi M=\pi M^{*} \subsetneq \pi L^{*} \subsetneq L \subsetneq \cdots
$$

with

$$
c(L)=0, \quad c\left(L^{*}\right)=-\frac{2}{3}, \quad c(M)=-\frac{1}{3} .
$$

Indeed, $L$ and $M$ are given by:

$$
\left\{\begin{array}{l}
L=\left\langle e_{1}, e_{2}, \pi e_{3}, e_{4}, \pi e_{-1}, \pi e_{-2}, e_{-3}, e_{-4}\right\rangle \\
M=\left\langle e_{1}, e_{2}, \pi e_{3}, e_{4}, e_{-1}, e_{-2}, \pi^{-1} e_{-3}, e_{-4}\right\rangle .
\end{array}\right.
$$

TOME $131-2003-\mathrm{N}^{\mathrm{O}} 3$ 
Moreover, since $v_{3}$ is a norm of algebra, the following are satisfied:

$$
\left\{\begin{array}{l}
L \text { is an order in } V, \text { and } \pi L^{*} \text { and } \pi M=\pi M^{*} \text { are ideals of } L \\
\left(\pi L^{*}\right)^{2} \subset \pi M \\
\left(\pi L^{*}\right) \cdot(\pi M) \subset \pi L \\
(\pi M)^{2} \subset \pi^{2} L^{*}
\end{array}\right.
$$

Note that $M$ is completely determined by $L$. Indeed, from (14), one can check that

$$
M=\pi L^{* 2}+L .
$$

Let $\mathcal{V}_{3}$ be the set of orders $L$ satisfying

$$
L \subsetneq L^{*} \subsetneq \pi^{-1} L, \quad M=\pi L^{* 2}+L \text { is a self-dual lattice. }
$$

Then we have shown that there is an injection from the set of vertices of type 3 into $\mathcal{V}_{3}$. Indeed we have:

Lemma 9.3. - The set of vertices of type 3 is in bijection with $\mathcal{V}_{3}$.

Proof. - This is similar to the proof above for vertices of type 2, though slightly more complicated. Given $L$, we assemble the graded lattice chain as in (12) and (13), and observe that the corresponding norm $\alpha$ is self-dual. We now need to check that $\alpha$ is an algebra norm, which is the same as checking the statements in (15). We do this systematically as follows:

- $L, L^{*}$ and $M$ are closed under conjugation. This is clear.

- $L^{*}$ is an ideal, i.e. $L \cdot L^{*} \subset L^{*}$ and $L^{*} \cdot L \subset L^{*}$. This follows from (11).

- $M$ is an ideal. This follows from (2).

- $L^{* 2} \subset \pi^{-1} M$. This follows from definition of $M$.

$-M \cdot L^{*} \subset \pi^{-1} L$ and $L^{*} \cdot M \subset \pi^{-1} L$. To see this, take any $x \in M$ and $y \in L^{*}$. For any $z \in L^{*}$, we have

$$
f(x \cdot y, \pi z)=f(x, \pi z \cdot \bar{y}) \in A,
$$

since $\pi z \cdot \bar{y} \in \pi L^{* 2} \subset M$. This implies that $M \cdot L^{*} \subset \pi^{-1} L$. The other containment follows similarly.

$-M^{2} \subset L^{*}$. If $x, y \in M$ and $z \in L$, then

$$
f(x \cdot y, z)=f(y, \bar{x} \cdot z) \in A,
$$

since $\bar{x} \cdot z \in M$.

We have thus shown that $\alpha$ is an algebra norm, which we can assume to lie in $C_{G}$. Moreover, the image of the norm $\alpha$ is $\frac{1}{3} \mathbb{Z}$, so that its coordinates $(a, b)$ lie in $\frac{1}{3} \mathbb{Z}$ but not both in $\mathbb{Z}$. There is now an added subtlety: there are two possible points in $C_{G}$ with this property, and their coordinates are

$$
(a, b)=\left(\frac{1}{3}, 0\right) \text { or }\left(0, \frac{1}{3}\right) \text {. }
$$

BULletin DE LA SOCiÉtÉ MATHÉmATiQUe DE FRANCE 
The first is the vertex $v_{3}$. After a computation, which we shall not reproduce here, one sees that the lattice chain corresponding to the second point is not of the above type. More precisely, the lattice chain corresponding to $\left(0, \frac{1}{3}\right)$ has the form (12) and satisfies (15), but it does not satisfy (16). Hence we have shown that $\alpha$ must be equal to $v_{3}$.

Corollary 9.4. - If $L \in \mathcal{V}_{3}$, then $L / \pi L^{*}$ is isomorphic to the étale algebra $A / \pi \times A / \pi$.

We have mentioned that a vertex of type 3 on $\mathcal{B}(G)$ is in fact the barycenter of a 3 -simplex in $\mathcal{B}(H)$ determined by 3 hyperspecial vertices. Given $L \in \mathcal{V}_{3}$, with corresponding lattice chain

$$
\cdots \subsetneq \pi L \subsetneq \pi M \subsetneq \pi L^{*} \subsetneq L \subsetneq \cdots
$$

one sees from (14) that $L / \pi L^{*} \cong A / \pi \times A / \pi$ is a split rank 2 quadratic space over the residue field $A / \pi$. As such, it has two isotropic lines. Let $N$ and $N^{\prime}$ be the inverse image of these isotropic lines in $L$. Then $N$ and $N^{\prime}$ satisfy $N=\pi N^{*}$ and $N^{\prime}=\pi N^{\prime *}$. The triple $\left\{M, N, N^{\prime}\right\}$ then determines 3 hyperspecial vertices of $\mathcal{B}(H)$ which form a 3 -simplex. The barycenter of this 3 -simplex is precisely the vertex of type 3 determined by $L$. Conversely, given $\left(M, N, N^{\prime}\right)$ as above, with $M$ self-dual, we have:

$$
L=N+N^{\prime}, \quad \pi L^{*}=N \cap N^{\prime} .
$$

We now summarize what we have proven as the first assertion of the following theorem.

THEOREM 9.5. - The vertices of $\mathcal{B}(G)$ of type $i$ are in natural bijection with the orders in $\mathcal{V}_{i}$. The incidence relation in $\mathcal{B}(G)$ can be described as follows.

(i) $L_{1}$ is incident to $L_{2}$ if and only if $L_{1} \supset L_{2}$.

(ii) $L_{1}$ is incident to $L_{3}$ if and only if $L_{1} \supset L_{3}$.

(iii) $L_{2}$ is incident to $L_{3}$ if and only if $L_{2} \supset L_{3}$.

(iv) $\left\{L_{1}, L_{2}, L_{3}\right\}$ is a chamber if and only if $L_{1} \supset L_{2} \supset L_{3}$.

Proof. - It remains to prove the statements about incidence relations. In each statement, the "only if" part is clear just by looking at the standard chamber $C_{G}$. We now prove the "if" part of (i). Without loss of generality, we may assume that $L_{1}$ corresponds to $v_{1} \in C_{G}$ and $L_{2}$ corresponds to a point on $\mathcal{A}(S)$.

To each point $\alpha \in \mathcal{B}(G)$, the lattice $L_{\alpha, 0}=\{x \in V: \alpha(x) \geq 0\}$ is an order in $V$. If $\alpha$ is on $\mathcal{A}(S)$ with coordinates $(a, b)$, we can use (7) and Lemma 6.3 to compute the condition $L_{\alpha, 0} \subset L_{1}$. It turns out that this condition is simply $-1<-a-b, a, 2 a+b<1$, i.e. $\alpha$ lies in the interior of the convex hull of the end points of the six long roots. It is clear from the figure that all vertices of type 2 in this region are incident to $v_{1}$. Hence (i) is proved. (ii) is proved in the same way.

TOME $131-2003-\mathrm{N}^{\mathrm{O}} 3$ 
(iii) is verified by a similar analysis. We may and do assume that $L_{2}$ corresponds to $v_{2} \in C_{G}$. For $\alpha \in \mathcal{A}(S)$, the condition $L_{\alpha, 0} \subset L_{2}$ is simply that $\alpha$ lies in the interior of the convex hull of $v_{1}, b, c, c-b$ (see the figure). It is then evident that every vertex of type 3 in this region is incident to $v_{2}$.

(iv) follows immediately from (i), (ii) and (iii).

COROllary 9.6. - The stabilizer in $G(k)$ of an order in $\mathcal{V}_{i}$ is a maximal parahoric subgroup of $G(k)$ fixing a vertex of type $i$.

REMARK 9.7. - The lattice chain associated to an order $L \in \mathcal{V}_{i}$ can be more conceptually described as follows. The quotient $\bar{L}=L / \pi L$ is an algebra over $A / \pi$, and the reduction $\bar{q}$ of $q$ is a quadratic form permitting composition. However, $\bar{q}$ may be degenerate. It is not difficult to see that its radical $\mathfrak{R}$ is an ideal of $\bar{L}$, and the quotient $\bar{L} / \Re$ is a composition algebra with non-degenerate norm form. Further, one has a finite decreasing sequence

$$
\mathfrak{R} \supset \mathfrak{R}^{2} \supset \mathfrak{R}^{3} \supset \cdots
$$

of ideals on $\bar{L}$. We call the inverse image of this sequence in $L$ the radical series of $L$. Then the lattice chain associated to $L$ is precisely its radical series.

Using the Cayley-Dickson construction, one can give a more natural description of the orders $L_{i}$. For simplicity, let us assume that $k$ is a local field. Let $B$ be a quaternion algebra over $k$, and fix a maximal order $R_{B}$ of $B$. If $\lambda$ is an element of $A$, then we have seen that one can construct an $A$-algebra $\operatorname{CD}\left(R_{B}, \lambda\right)$, which is an order in $\operatorname{CD}(B, \lambda) \otimes k \cong V$. Now we have:

Proposition 9.8. - (i) If $B$ is split, and $\lambda$ is a unit, then $\operatorname{CD}\left(R_{B}, \lambda\right)$ is a maximal order in $V$, and thus is isomorphic to $L_{1}$.

(ii) If $B$ is split, and $\lambda=\pi$, then $\operatorname{CD}\left(R_{B}, \lambda\right)$ is isomorphic to $L_{2}$.

(iii) If $B$ is non-split, and $\lambda$ is a unit, then $\operatorname{CD}\left(R_{B}, \lambda\right)$ is isomorphic to $L_{2}$.

(iv) Suppose that $B$ is split and let $R(\pi)$ be an order contained in $R_{B}$ with $R_{B} / R(\pi) \cong A / \pi$. If $\lambda$ is a unit, then $\operatorname{CD}(R(\pi), \lambda)$ is isomorphic to $L_{2}$.

(v) With the notations of (iv), let $J(\pi)$ be the two-sided ideal of $R(\pi)$ with $R(\pi) / J(\pi) \cong A / \pi$. If $\lambda$ is a unit, then $\operatorname{CD}(R(\pi), J(\pi), \lambda)$ is isomorphic to $L_{3}$.

Proof. - In each case, one verifies directly that the order in question lies in the relevant set $\mathcal{V}_{i}$. The result then follows by the first assertion of Theorem 9.5. We omit the details.

REMARK 9.9. - The above proposition shows that $R_{B}$ always embeds as a subring of $L_{1}$ or $L_{2}$. On the other hand, $R_{B}$ cannot be embedded as a subring of $L_{3}$. To see this, observe that the $A / \pi$-algebra $L_{3} / \pi L_{3}$ has a 6 -dimensional two-sided ideal, for which the corresponding quotient ring is isomorphic to $A / \pi \times A / \pi$. However, it is easy to see that $R_{B}$ does not possess such a quotient ring.

BULletin DE LA SOCIÉtÉ MATHÉMATIQUE DE FRANCE 
It is an instructive exercise to recover the usual description [1] of the spherical building of type $G_{2}$ from the description of the simplicial structure of $\mathcal{B}(G)$ given above. For this, let us fix the hyperspecial vertex $v_{1}$, which corresponds to the maximal order $R$. Then the link of $v_{1}$ in $\mathcal{B}(G)$ is the spherical building of $\underline{G} \times A / \pi$. Suppose we are given a vertex of type 2 which is incident to $v_{1}$. Let $L_{2}$ be the corresponding order. Then $L_{2} / \pi R$ is a 6 -dimensional subspace of $R / \pi R$, and its orthogonal complement is the 2-dimensional subspace $\pi L_{2}^{*} / \pi R$, on which the octonion multiplication is trivial, and all of whose elements have trace zero. As is well-known, the stabilizer of such a 2dimensional subspace in $R / \pi R$ is a (Heisenberg) maximal parabolic subgroup of $\underline{G} \times A / \pi$. Conversely, it is easy to recover the lattice $L_{2}$ if we are given such a 2-dimensional subspace of $R / \pi R: \pi L_{2}^{*}$ is simply the inverse image of this subspace in $R$. Now suppose $L_{3}$ is an order in $V_{3}$ whose corresponding vertex is incident to $v_{1}$. Then $L_{3}$ gives rise to a self-dual lattice $M$ as in (16), and $(\pi M+\pi R) / \pi R$ is a 1 -dimensional subspace of $R / \pi R$ consisting of trace zero elements and on which the octonion multiplication is trivial. It is well-known that the stabilizer of such a subspace of $R / \pi R$ is a maximal parabolic subgroup of $\underline{G} \times A / \pi$ belonging to the other conjugacy class. Conversely, if we are given such a 1-dimensional subspace $\langle x\rangle$ of $R / \pi R$, let

$$
x \Delta=\{y \in R / \pi R: x \cdot y=y \cdot x=0\} .
$$

Then $x \Delta$ is 3 -dimensional and its inverse image in $R$ is the lattice $\pi L_{3}^{*}$. From this, we can easily recover $L_{3}$.

\section{Group Schemes}

The smooth group schemes associated to the parahoric subgroups can be described using the orders in $\mathcal{V}:=\mathcal{V}_{1} \cup \mathcal{V}_{2} \cup \mathcal{V}_{3}$ :

TheOREM 10.1. - Given an order $L$ in $\mathcal{V}$, the schematic closure of $G$ in $\operatorname{Aut}(L)$ is the smooth integral model $\underline{G}_{x}$ of $G$ associated to the vertex $x$ corresponding to $L$. More generally, let $X$ be a finite set of vertices and $\mathcal{L} \subset \mathcal{V}$ be the set of orders corresponding to elements of $X$. Assume that there is an apartment $\mathcal{A}$ on $\mathcal{B}(G)$ such that $X \subset \mathcal{A}$ and let $\Omega \subset \mathcal{A}$ be the convex hull of $X$. Then the schematic closure of $G$ in $\prod_{L \in \mathcal{L}} \operatorname{Aut}(L)$ is the smooth integral model $\underline{G}_{\Omega}$ of $G$ associated to $\Omega[17,3.4 .1]$.

We recall $[17,3.4 .1]$ that $\underline{G}_{\Omega}$ is characterized by

(a) $\underline{G}_{\Omega}$ is affine smooth group scheme over $A$ with generic fiber $G$;

(b) for any unramified extension $\widetilde{k} / k, \underline{G}_{\Omega}(\widetilde{A})$ is the subgroup of $G(\widetilde{k})$ consisting of elements fixing $X$ pointwise, where $\widetilde{A}$ is the ring of integers in $\widetilde{k}$.

Let $\underline{G}$ be the schematic closure of $G$ in $\prod_{L \in \mathcal{L}} \operatorname{Aut}(L)$. It is clear that $\underline{G}$ is an affine group scheme of finite type over $A$ satisfying (b). The key point is to

TOME $131-2003-\mathrm{N}^{\mathrm{O}} 3$ 
show that $\underline{G}$ is smooth, and the verification will occupy much of the remainder of this section.

Let $S$ be the maximal $k$-split torus corresponding to $\mathcal{A}$ and $\left\{U_{b}\right\}_{b \in \Phi(G, S)}$ be the root subgroups of $G$ relative to $S$. By [4, 2.2.5], to prove the smoothness of $\underline{G}$, it suffices to show the following two assertions

(i) the schematic closure $\underline{S}$ of $S$ in $\prod_{L \in \mathcal{L}} \operatorname{Aut}(L)$ is smooth;

(ii) for each $b \in \Phi(G, S)$, the schematic closure $\underline{U}_{b}$ of $U_{b}$ in $\prod_{L \in \mathcal{L}} \operatorname{Aut}(L)$ is smooth.

Assertion (i) is clear from the assumption $X \subset \mathcal{A}$. It remains to prove assertion (ii). This will be divided into two cases, depending on whether $b$ is a long root or a short root.

Suppose that $b$ is a long root. Then $G_{b}=\left\langle U_{b}, S, U_{-b}\right\rangle$ is isomorphic to $\mathrm{GL}_{2}$. Regarding $V$ as a representation of $G_{b} \simeq \mathrm{GL}_{2}$, we have a decomposition into isotypical subspaces $V=\bigoplus_{i=1}^{5} V_{i}$, with

$$
V_{1} \simeq \mathbf{1}^{\oplus 2}, \quad V_{2} \simeq \operatorname{det}, \quad V_{3} \simeq(\operatorname{det})^{\vee}, \quad V_{4} \simeq \mathrm{st}, \quad V_{5} \simeq \mathrm{st}^{\vee} .
$$

Here, st denotes the standard representation of $\mathrm{GL}_{2}$. It is easy to see that for each $L \in \mathcal{L}, L=\bigoplus_{i=1}^{5} L \cap V_{i}$. Since $\prod_{L \in \mathcal{L}} \prod_{i=1}^{5} \operatorname{Aut}\left(L \cap V_{i}\right)$ is a closed sub-scheme of $\prod_{L \in \mathcal{L}} \operatorname{Aut}(L)$ and $U_{b} \rightarrow \prod_{L \in \mathcal{L}} \mathrm{GL}(V)$ factors through $\prod_{L \in \mathcal{L}} \prod_{i=1}^{5} \mathrm{GL}\left(V_{i}\right)$, It follows that $\underline{U}_{b}$ is the schematic closure of $U_{b}$ in $\prod_{L \in \mathcal{L}} \prod_{i=1}^{5} \operatorname{Aut}\left(L \cap V_{i}\right)$, and is also the schematic closure of $U_{b}$ in $\prod_{L \in \mathcal{L}} \prod_{i=4}^{5} \operatorname{Aut}\left(L \cap V_{i}\right)$.

LEMMA 10.2. - Let $W$ be the standard representation of $H=\mathrm{GL}_{2}$. Let $\mathcal{A}=$ $\mathcal{A}(H, S)$ be an apartment on $\mathcal{B}(H)$ corresponding to the maximal $k$-split torus $S$ of $H$. Let $X$ and $X^{\prime}$ be two finite subsets of hyperspecial points on $\mathcal{A}$. For $x \in X \cup X^{\prime}$, let $M_{x} \subset W, M_{x}^{\vee} \subset W^{\vee}$ be the lattices whose stabilizer is $H(k)_{x}$. Let $\Omega$ be the convex hull of $X \cup X^{\prime}$. Then

(i) The schematic closure of $H$ in $\prod_{x \in X} \operatorname{Aut}\left(M_{x}\right) \times \prod_{x \in X^{\prime}} \operatorname{Aut}\left(M_{x}^{\vee}\right)$ is the smooth integral model $\underline{H}_{\Omega}$ of $H$ associated to $\Omega$.

(ii) For $b \in \Phi(H, S)$, the schematic closure of $U_{b} \subset H$ in $\prod_{x \in X} \operatorname{Aut}\left(M_{x}\right) \times$ $\prod_{x \in X^{\prime}} \operatorname{Aut}\left(M_{x}^{\vee}\right)$ is smooth.

Proof. - It is clear that $\operatorname{Aut}\left(M_{x}^{\vee}\right)=\operatorname{Aut}\left(M_{x}\right)$. Therefore, we may and do assume that $X^{\prime}=\varnothing$. Then (i) and (ii) are just special cases of $[5,3.9 .2]$.

We now return to the proof of the smoothness of $\underline{U}_{b}$ for a long root $b$. For $L \in \mathcal{L}$, the lattice $L \cap V_{4}$ corresponds to a vertex $x_{L}$ on $\mathcal{A}\left(G_{b}, S\right) \subset \mathcal{B}\left(G_{b}\right)$ by [5]. Similarly, the lattice $L \cap V_{5}$ corresponds to a vertex $x_{L}^{\prime}$ on $\mathcal{A}\left(G_{b}, S\right)$. It is now clear that the smoothness of $\underline{U}_{b}$ follows from part (ii) of Lemma 10.2.

Now suppose that $b \in \Phi(G, S)$ is a short root. Then $G_{b}=\left\langle U_{b}, S, U_{-b}\right\rangle$ is again isomorphic to $\mathrm{GL}_{2}$. Regarding $V$ as a representation of $G_{b} \simeq \mathrm{GL}_{2}$, we 
have $V \simeq V_{1} \oplus V_{2} \oplus V_{3}$, with

$$
V_{1} \simeq \mathrm{st} \otimes \mathrm{st}^{\vee}, \quad V_{2}=\mathrm{st}, \quad V_{3}=\mathrm{st}^{\vee} .
$$

Again it is easy to see that $L=\bigoplus_{i=1}^{3} L \cap V_{i}$ for each $L \in \mathcal{L}$.

It is easy to show that $V_{1}$ is a sub-algebra of $V$ isomorphic to $M_{2}(k)$. We can write down an isomorphism $\phi: V_{1} \simeq M_{2}(k)$ such that $\phi\left(V_{1}^{S}\right)$ is the subset of diagonal matrices, and $L \cap V_{1}$ is an order of the form

$$
R_{n}=\left\{\left(\begin{array}{cc}
a & b \\
\pi^{n} c & d
\end{array}\right) \mid a, b, c, d \in A\right\}, \quad n=0 \text { or } 1 .
$$

LEMma 10.3. - Let $W$ be the standard representation of $H=\mathrm{GL}_{2}$ and $S \subset H$ be the maximal $k$-split torus consisting of diagonal elements. Let $R_{n}$ be as above with $n \geq 0$. Then there exist lattices $M, M^{\prime} \subset W$ corresponding to points $x, x^{\prime}$ in $\mathcal{A}(H, S)$ such that the schematic closure of $U_{b}$ in $\operatorname{Aut}\left(R_{n}\right)$ is the same as the schematic closure of $U_{b}$ in $\operatorname{Aut}(M) \times \operatorname{Aut}\left(M^{\prime}\right)$ for each root subgroup $U_{b}$ of $H$ relative to $S$.

Proof. - Let $w_{1}, w_{2}$ be the standard basis of $W$, then it is easy to check that $M=\left\langle w_{1}, w_{2}\right\rangle, M^{\prime}=\left\langle w_{1}, \pi^{n} w_{2}\right\rangle$ fulfill the conditions of the lemma.

Now for each $L \in \mathcal{L}$, we have a lattice $L^{(1)}=L \cap V_{2}$ in $W$, a lattice $L^{(2)}=L \cap V_{3}$ in $W^{\vee}$, two lattices $L^{(3)}=M$ and $L^{(4)}=M^{\prime}$ in $W$ associated to $L \cap V_{1}$ by the above lemma. All the lattices $\left\{L^{(i)}: L \in \mathcal{L}, i=1,2,3,4\right\}$ correspond to points in $\mathcal{A}\left(G_{b}, S\right)$, and $\underline{U}_{b}$ is the schematic closure of $U_{b}$ in $\prod_{L \in \mathcal{L}} \prod_{i=1}^{4} \operatorname{Aut}\left(L^{(i)}\right)$. Now part (ii) of Lemma 10.2 again implies that $\underline{U}_{b}$ is smooth.

Thm. 10.1 has been proved completely.

Let $L$ be an order in $\mathcal{V}$, corresponding to a vertex $x$, with associated graded lattice chain $\left(L_{x, r}\right)$ and smooth group scheme $\underline{G}_{x}$. Thm. 10.1 implies that $\underline{G}_{x} \times A / \pi$ is a closed subgroup scheme of $\operatorname{Aut}(L / \pi L)$, and thus acts on the vector space $L / \pi L$ over $A / \pi$. The rest of this section is devoted to a study of this rational representation of the special fiber.

Let $\bar{G}$ be the maximal reductive quotient of the special fiber $\underline{G}_{x} \times A / \pi$. The isomorphism class of $\bar{G}$ is known by Bruhat-Tits theory [17]. Indeed,

$$
\bar{G} \cong \begin{cases}\underline{G} \times A / \pi, & \text { if } x \in \mathcal{V}_{1} \\ \left(\mathrm{SL}_{2} \times \mathrm{SL}_{2}\right) / \Delta \mu_{2} \cong \mathrm{SO}_{4}, & \text { if } x \in \mathcal{V}_{2} \\ \mathrm{SL}_{3}, & \text { if } x \in \mathcal{V}_{3}\end{cases}
$$

Further, Bruhat-Tits theory associates to the vertex $x$ a decreasing filtration $\left(G_{x, r}\right)_{r \geq 0}$ of open compact subgroups of $G_{x, 0}=\underline{G}_{x}(A)$, such that

$$
G_{x, 0} / G_{x, 1} \cong \underline{G}_{x}(A / \pi), \quad G_{x, 0} / G_{x, 0+} \cong \bar{G}(A / \pi) .
$$

TOME $131-2003-\mathrm{N}^{\mathrm{O}} 3$ 
Since $\underline{G}_{x}(A)$ stabilizes each of the lattices $L_{x, r}$, we see that for $0 \leq r \leq 1$, $L_{x, r} / \pi L$ is a sub-representation of $L / \pi L$. One thus has the sub-quotient representations $L_{x, r} / L_{x, r+}$ of the special fiber. It is not difficult to show that, in fact, $G_{x, 0+}$ acts trivially on $L_{x, r} / L_{x, r+}$, which then implies that the action of the special fiber on $L_{x, r} / L_{x, r+}$ factors through $\bar{G}$. The following proposition describes these representations of $\bar{G}$ explicitly.

Proposition 10.4. - (i) If $x \in \mathcal{V}_{1}, L / \pi L$ is the split octonion algebra over $A / \pi$, which is the standard representation of $\bar{G} \cong \operatorname{Aut}(L / \pi L)$.

(ii) If $x \in \mathcal{V}_{2}$, the filtration

$$
0 \subset \pi L^{*} / \pi L \subset L / \pi L
$$

has successive quotients, which, as representations of $\bar{G}$, are given by

$$
L / \pi L^{*} \cong \operatorname{ad} \otimes \mathbf{1}, \quad L^{*} / L \cong \text { standard representation of } \mathrm{SO}_{4},
$$

where ad is the adjoint action of $\mathrm{SL}_{2}$ on the space of $2 \times 2$ matrices over $A / \pi$.

(iii) If $x \in \mathcal{V}_{3}$, the filtration

$$
0 \subset \pi M / \pi L \subset \pi L^{*} / \pi L \subset L / \pi L
$$

has successive quotients, which, as representations of $\bar{G}$, are given by

$$
L / \pi L^{*} \cong \mathbf{1}^{\oplus 2}, \quad L^{*} / M \cong \mathrm{st}, \quad M / L \cong \mathrm{st}^{*},
$$

where st is the standard representation of $\bar{G}$, under a suitable identification with $\mathrm{SL}_{3}$.

Proof. - Assertion (i) is a direct consequence of the considerations in $\S 6$, and in particular Cor.6.2. Since (ii) and (iii) are proved in similar ways, we will only give the proof for (ii).

Without loss of generality, we assume that $x=v_{2}$, so that $L=L_{2}$. Let $\bar{S}=\underline{S} \times A / \pi$, where we recall that $S$ is a maximal $k$-split torus of $G$. Then we have an embedding

$$
\bar{S} \hookrightarrow \bar{G} \simeq\left(\mathrm{SL}_{2} \times \mathrm{SL}_{2}\right) / \Delta \mu_{2} \simeq \mathrm{SO}_{4}
$$

of algebraic groups over $A / \pi$. The root subgroups of $\bar{G}$ relative to $\bar{S}$ are special fibers of the root subgroups of $\underline{G}_{x}$ corresponding to the roots $\pm a$ and $\pm \delta=$ $\pm(3 a+2 b)$. We adopt the convention that the first copy of $\mathrm{SL}_{2}$ in $\bar{G}$ is the one associated to $\pm a$.

Now the weights of $S$ on $V$ are precisely the 6 short roots and the trivial character with multiplicity 2. Using Lemma 6.3 and the explicit basis for $L=L_{2}$ given in the previous section, it is easy to see that the weights of $\bar{S}$ on $L / \pi L^{*}$ are $\pm a$ and the trivial character with multiplicity 2 . From this, one deduces that as a representation of $\bar{G}$,

$$
L / \pi L^{*} \simeq \operatorname{ad} \otimes \mathbf{1}
$$

BULletin DE LA SOCiÉtÉ MATHÉMATIQUE DE FRANCE 
On the other hand, the weights of $\bar{S}$ on $L^{*} / L$ are $\pm(a+b)$ and $\pm(2 a+b)$, from which it follows that

$$
L^{*} / L \simeq \text { standard representation of } \mathrm{SO}_{4}
$$

as a representation of $\bar{G}$.

\section{Triality}

The closed embedding $\iota: G \hookrightarrow \mathrm{SO}(V)$ can be lifted to a closed embedding $\widetilde{\iota}: G \hookrightarrow \operatorname{Spin}(V)$, where $\operatorname{pr}_{1}: \operatorname{Spin}(V) \rightarrow \mathrm{SO}(V)$ is the simply-connected cover of $H=\mathrm{SO}(V)$. There is a $S_{3^{-}}$action on $\operatorname{Spin}(V)$ whose group of fixed points is precisely $G$. This induces an action of $S_{3}$ on the building $\mathcal{B}(\operatorname{Spin}(V))$, which can be naturally identified with the building $\mathcal{B}(H)$ using $\mathrm{pr}_{1}$. We already know that $\mathcal{B}(G)$ is a subset of $\mathcal{B}(H)$, and hence of $\mathcal{B}(\operatorname{Spin}(V))$. It is thus natural to ask if the set of fixed points of $\mathcal{B}(\operatorname{Spin}(V))$ under this $S_{3}$-action is equal to $\mathcal{B}(G)$. When $p \neq 2,3$, the general result of [13] shows that it is indeed the case. In this section, we give a proof which works for all $p$, and in the process, we give an explicit description of the building $\mathcal{B}(\operatorname{Spin}(V))$, together with the action of $S_{3}$.

We begin with a more concrete description of the group $\operatorname{Spin}(V)$ over $\mathbb{Z}$. Recall that we have introduced in Section 5 the octonion algebra $(\Lambda, q, \cdot)$ over $\mathbb{Z}$. It is now more convenient to work with the multiplication $*$ on $\Lambda$ instead of the octonion multiplication. Recall that the two are related by: $x * y=\bar{x} \cdot \bar{y}$. The self-dual lattice $\Lambda$ can be used to define the Chevalley group $\operatorname{Spin}(\Lambda)$ with generic fiber $\operatorname{Spin}(V)$ as follows. For any $\mathbb{Z}$-algebra $B$, we have:

$\operatorname{Spin}(\Lambda)(B)=\left\{\left(g_{1}, g_{2}, g_{3}\right) \in \underline{H}(B)^{3}: g_{1}(x * y)=g_{2}(x) * g_{3}(y), \forall x, y \in \Lambda \otimes B\right\}$. This realizes $\operatorname{Spin}(\Lambda)$ as a subgroup of $\underline{H}^{3}$, and we let

$$
\delta: \operatorname{Spin}(\Lambda) \longleftrightarrow \underline{H}^{3}
$$

be the closed immersion. The three projections then give the three central isogenies

$$
\operatorname{pr}_{1}, \operatorname{pr}_{2}, \operatorname{pr}_{3}: \operatorname{Spin}(\Lambda) \longrightarrow \underline{H} \text {. }
$$

Let $\underline{T}$ be the maximal torus of $\underline{H}$ constructed in Section 6 . Then $\underline{T}^{3}$ is a maximal split torus of $\underline{H}^{3}$, and $\underline{T}^{3} \cap \operatorname{Spin}(\Lambda)$ is a maximal split torus of $\operatorname{Spin}(\Lambda)$.

We have constructed an embedding $\iota: \underline{G} \hookrightarrow \underline{H}$ in Section 6 . Using $\iota^{3}$, we embed $\underline{G}$ as a subgroup of $\underline{H}^{3}$. Since $\underline{G}$ is also the automorphism group of the multiplication $*[10$, Remark 34.7], it is clear that the embedding factors through the subgroup $\operatorname{Spin}(\Lambda)$, so that we have an embedding $\widetilde{\iota}: \underline{G} \hookrightarrow \operatorname{Spin}(\Lambda)$. Moreover, for $i=1,2$ or 3 , we have:

$$
\operatorname{pr}_{i} \circ \delta \circ \widetilde{\iota}=\iota
$$

as morphisms from $\underline{G}$ to $\underline{H}$.

TOME $131-2003-\mathrm{N}^{\mathrm{O}} 3$ 
We now describe an action of the finite group $S_{3}$ on $\underline{H}^{3}$ which restricts to an action on the subgroup $\operatorname{Spin}(\Lambda)$. Let the group $\mathbb{Z} / 3 \mathbb{Z}$ act on $\underline{H}^{3}$ by cyclic permutation. Further, for $g \in \underline{H}$, let $\hat{g}$ be the automorphism of $\Lambda$ given by

$$
\hat{g}: x \longmapsto \overline{g(\bar{x})} \text {. }
$$

Then $\hat{g}$ is also an element of $\underline{H}$ and we define an involution on $\underline{H}^{3}$ by:

$$
\left(g_{1}, g_{2}, g_{3}\right) \longmapsto\left(\hat{g}_{1}, \hat{g}_{3}, \hat{g}_{2}\right) \text {. }
$$

This involution and the $\mathbb{Z} / 3 \mathbb{Z}$-action above generate an action of $S_{3}$ on $\underline{H}^{3}$. This preserves the subgroup $\operatorname{Spin}(\Lambda)$, and it is clear that

$$
\operatorname{Spin}(\Lambda)^{S_{3}}=\operatorname{Spin}(\Lambda)^{\mathbb{Z} / 3 \mathbb{Z}}=\underline{G} .
$$

By base extension, we have the group $\operatorname{Spin}(V)$ over $k$, together with the closed embeddings

$$
\delta: \operatorname{Spin}(V) \longleftrightarrow H^{3}, \quad \widetilde{\iota}: G \longleftrightarrow \operatorname{Spin}(V) .
$$

Being assembled from central isogenies, $\delta$ can be canonically completed to a strong descent datum $\left(\delta_{E *}\right)$. It follows from definition that the induced action of $\mathbb{Z} / 3 \mathbb{Z}$ on $\mathcal{B}(H)^{3}$ is by cyclic permutation, and this restricts to the induced action of $\mathbb{Z} / 3 \mathbb{Z}$ on $\mathcal{B}(\operatorname{Spin}(V))$. Further, the induced action of the involution $g \mapsto \hat{g}$ on $\mathcal{B}(H)^{3}$ is given by

$$
\left(\alpha_{1}, \alpha_{2}, \alpha_{3}\right) \longmapsto\left(\bar{\alpha}_{1}, \bar{\alpha}_{3}, \bar{\alpha}_{2}\right),
$$

where $\bar{\alpha}$ is the norm defined by $\bar{\alpha}(x)=\alpha(\bar{x})$, and this restricts to the induced action of the involution on $\mathcal{B}(\operatorname{Spin}(V))$. Thus $\delta_{E *}$ is $S_{3}$-equivariant. Moreover, it follows by construction that for $i=1,2,3$, we have:

$$
\operatorname{pr}_{i *} \circ \delta_{*} \circ \widetilde{\iota}_{*}=\iota_{*}
$$

as maps from $\mathcal{B}(G)$ to $\mathcal{B}(H)$.

The adjoint group of $\operatorname{Spin}(V)$ is $\operatorname{PGSO}(V)$, and $\operatorname{PGSO}(V)(k)$ acts naturally on $\mathcal{B}(\operatorname{Spin}(V))$ and $\mathcal{B}(H)$. We first describe the action of $\operatorname{PGSO}(V)(k)$ on $\mathcal{B}(H)$. Let $g_{1} \in \operatorname{GSO}(V)(k)$, with similitude factor $\lambda\left(g_{1}\right)$. Then, for $\alpha_{1} \in \mathcal{B}(H)$, we set:

$$
\left(g_{1} \alpha_{1}\right)(x)=\alpha_{1}\left(g_{1}^{-1}(x)\right)+\frac{1}{2} \operatorname{ord}\left(\lambda\left(g_{1}\right)\right) .
$$

This depends only on the image of $g_{1}$ in $\operatorname{PGSO}(V)(k)$, and defines the action of $\operatorname{PGSO}(V)(k)$ on $\mathcal{B}(H)$. The action of $\operatorname{PGSO}(V)(k)$ on $\mathcal{B}(\operatorname{Spin}(V))$ can be described using the embedding $\delta_{*}$. We first note [10, Prop. 35.4]:

Proposition 11.1. - Given arbitrary $g_{1} \in \operatorname{GSO}(V)(k)$ with similitude factor $\lambda\left(g_{1}\right)$, there exist $g_{2}, g_{3} \in \operatorname{GSO}(V)(k)$, with similitude factors $\lambda\left(g_{2}\right)$ and $\lambda\left(g_{3}\right)$, such that

$$
\lambda\left(g_{i}\right)^{-1} g_{i}(x * y)=g_{i+1}(x) * g_{i+2}(y), \quad \text { for all } x, y \in V,
$$

BULletin DE LA SOCiÉtÉ MATHÉMATIQUE DE FRANCE 
where $i$ is taken modulo 3. Moreover,

$$
\lambda\left(g_{1}\right) \lambda\left(g_{2}\right) \lambda\left(g_{3}\right)=1,
$$

and the pair $\left(g_{2}, g_{3}\right)$ is well-defined up to scaling by $\left(\lambda, \lambda^{-1}\right)$. In particular, as elements of $\operatorname{PGSO}(V)(k), g_{2}$ and $g_{3}$ are uniquely determined by $g_{1}$.

Consider the algebraic subgroup $H^{\prime}$ of $\operatorname{PGSO}(V)^{3}$ consisting of $\left(g_{1}, g_{2}, g_{3}\right)$ satisfying the conditions in Prop. 11.1. The proposition implies that we have an isomorphism

$$
\operatorname{PGSO}(V) \longrightarrow H^{\prime}
$$

given by

$$
g_{1} \longmapsto\left(g_{1}, g_{2}, g_{3}\right),
$$

whose inverse is equal to the first projection. Now $H^{\prime}(k)$ acts on $\mathcal{B}(H)^{3}$ by the formula (18):

$$
\left(g_{1}, g_{2}, g_{3}\right):\left(\alpha_{1}, \alpha_{2}, \alpha_{3}\right) \longmapsto\left(g_{1} \alpha_{1}, g_{2} \alpha_{2}, g_{3} \alpha_{3}\right),
$$

and its restriction to the subset $\mathcal{B}(\operatorname{Spin}(V))$ is the action of $\operatorname{PGSO}(V)(k)$ on $\mathcal{B}(\operatorname{Spin}(V))$. In other words, the embedding $\delta_{*}$ is equivariant with respect to the action of $\operatorname{PGSO}(V)(k)$ on $\mathcal{B}(\operatorname{Spin}(V))$ and that of $H^{\prime}(k)$ on $\mathcal{B}(H)^{3}$.

We can now determine the image of $\delta_{*}$ by applying the formalism given in Thm. 3.5. Regarding $\mathcal{B}(H)^{3}$ as the set of triples of maximinorante norms on $V$, we let $\mathcal{N}_{E}$, for any finite extension $E$ of $k$, be the subset of $\mathcal{B}\left(H_{E}\right)^{3}$ given by:

$$
\mathcal{N}_{E}=\left\{\left(\alpha_{1}, \alpha_{2}, \alpha_{3}\right) \in \mathcal{B}\left(H_{E}\right)^{3}: \alpha_{1}(x * y) \geq \alpha_{2}(x)+\alpha_{3}(y) \text { for all } x, y \in V\right\} .
$$

It is interesting to note the following lemma:

LEMMA 11.2. - The following properties of the triple $\left(\alpha_{1}, \alpha_{2}, \alpha_{3}\right)$ of self-dual norms are equivalent:

(i) $\alpha_{1}(x * y) \geq \alpha_{2}(x)+\alpha_{3}(y)$ for all $x, y \in V$.

(ii) $\alpha_{i}(x * y) \geq \alpha_{i+1}(x)+\alpha_{i+2}(y)$ for all $x, y \in V$, and for $i=1,2,3$.

(iii) $\operatorname{ord}((x, y, z)) \geq \alpha_{1}(\bar{x})+\alpha_{2}(\bar{y})+\alpha_{3}(\bar{z})$, for all $x, y, z \in V$, and where $(x, y, z)=\operatorname{Tr}(x y z)$.

Proof. - Suppose that $\left(\alpha_{1}, \alpha_{2}, \alpha_{3}\right)$ satisfies (i). We shall show that so does $\left(\alpha_{2}, \alpha_{3}, \alpha_{1}\right)$, which will be sufficient to prove (ii). Since $\alpha_{2}$ is self-dual with respect to $f$, for any given $x$ and $y$, there exists $z$ such that

$$
\alpha_{2}(x * y)=\operatorname{ord} f(x * y, z)-\alpha_{2}(z) .
$$

Since $f(x * y, z)=f(z, x * y)=f(z * x, y)$, we see that

$$
\begin{aligned}
\alpha_{2}(x * y) & =\operatorname{ord} f(z * x, y)-\alpha_{2}(z) \\
& \geq \alpha_{1}(z * x)+\alpha_{1}(y)-\alpha_{2}(z) \geq \alpha_{3}(x)+\alpha_{1}(y),
\end{aligned}
$$

as desired. 
It is easy to show that (ii) implies (iii). So let us now assume that (iii) holds and proves (i). By the self-duality of $\alpha_{1}$, for any given $x$ and $y$, there exists $z$ such that

$$
\alpha_{1}(x * y)=\operatorname{ord} f(x * y, z)-\alpha_{1}(z) .
$$

By (iii), we see that

$$
\begin{aligned}
\alpha_{1}(x * y) & =\operatorname{ord}(\bar{x}, \bar{y}, \bar{z})-\alpha_{1}(z) \\
& =\operatorname{ord}(\bar{z}, \bar{x}, \bar{y})-\alpha_{1}(z) \geq \alpha_{2}(\bar{x})+\alpha_{3}(\bar{y}) .
\end{aligned}
$$

The lemma is proved.

Now we have:

THEOREM 11.3. - The image of $\delta_{*}$ is the subset $\mathcal{N}$. Via $\delta_{*}$, the building $\mathcal{B}(\operatorname{Spin}(V))$ is identified with the set $\mathcal{N}$ of triples of norms $\left(\alpha_{1}, \alpha_{2}, \alpha_{3}\right)$ on $V$ which are maximinorante for $(q, f)$, and which satisfy

$$
\alpha_{1}(x * y) \geq \alpha_{2}(x)+\alpha_{3}(y) .
$$

The $S_{3}$-action on $\mathcal{B}(\operatorname{Spin}(V))$ is generated by

$$
\left(\alpha_{1}, \alpha_{2}, \alpha_{3}\right) \mapsto\left(\alpha_{3}, \alpha_{1}, \alpha_{2}\right)
$$

and

$$
\left(\alpha_{1}, \alpha_{2}, \alpha_{3}\right) \mapsto\left(\bar{\alpha}_{1}, \bar{\alpha}_{3}, \bar{\alpha}_{2}\right)
$$

Proof. - Once again, we shall make use of Thm.3.5. Conditions (BC) and (RAT) for $\left\{\mathcal{N}_{E}\right\}$ can be easily checked; we omit the details.

It remains to verify the condition (TRANS). Let $y_{0}=\left(x_{0}, x_{0}, x_{0}\right) \in \mathcal{B}(H)^{3}$, where $x_{0}$ is the hyperspecial point on $\mathcal{B}(H)$ as in Thm. 7.2. Recall that $x_{0}$ gives rise to the lattice chain of rank 1 determined by the maximal order $R$ of $V$. By (17), we see that

$$
y_{0} \in \mathcal{N} \cap\left(\delta_{*} \circ \widetilde{\iota}_{*}(\mathcal{B}(G))\right) \subset \mathcal{N} \cap \delta_{*}(\mathcal{B}(\operatorname{Spin}(V)),
$$

and is moreover the image of a rational point on $\mathcal{B}(\operatorname{Spin}(V))$. Hence, the first condition in (TRANS) holds with the rational point $y_{0}$.

We now check the second condition in (TRANS). Suppose that $y=\left(\alpha_{1}, \alpha_{2}\right.$, $\left.\alpha_{3}\right) \in \mathcal{N}_{E}$ is in the $H(E)^{3}$-orbit of $y_{0}$. In particular, $\alpha_{i}$ is a hyperspecial vertex of $\mathcal{B}\left(H_{E}\right)$; in fact, each of them corresponds to a self-dual lattice. We need to show that $y$ is in the image of $\delta_{E *}$. Now the map

$$
\delta_{E *}: \mathcal{B}\left(\operatorname{Spin}\left(V_{E}\right)\right) \longleftrightarrow \mathcal{B}\left(H_{E}\right)^{3},
$$

is not only $\operatorname{Spin}(V)(E)$-equivariant, but also $H^{\prime}(E)$-equivariant. Hence it suffices to show that $y$ is in the $H^{\prime}(E)$-orbit of $y_{0}$.

BULLETIN DE LA SOCIÉtÉ MATHÉMATIQUE DE FRANCE 
Let $g_{1} \in H(E)$ be such that $x_{0}=g_{1} \alpha_{1}$ and let $g=\left(g_{1}, g_{2}, g_{3}\right) \in H^{\prime}(E)$. It is easy to check that $H^{\prime}(E)$ preserves $\mathcal{N}_{E}$, and hence the point $g \cdot y=$ $\left(x_{0}, g_{2} \alpha_{2}, g_{3} \alpha_{3}\right)$ lies in $\mathcal{N}_{E}$. Now $g_{2} \alpha_{2}$ and $g_{3} \alpha_{3}$ are hyperspecial vertices of $\mathcal{B}(H)$ but they may not correspond to self-dual lattices now, since $g_{2}$ and $g_{3}$ may not be in $H(E)$. More precisely, they correspond either to self-dual lattices or to lattices $L$ with $L=\pi_{E} L^{*}$.

Now let

$$
L=\left\{x \in V:\left(g_{2} \alpha_{2}\right)(x) \geq 0\right\}, \quad M=\left\{x \in V:\left(g_{3} \alpha_{3}\right)(x) \geq 0\right\} .
$$

The fact that $g \cdot y \in \mathcal{N}_{E}$ implies, in view of Lemma 11.2, that

$$
R_{E} * L \subset M \text { and } M * R_{E} \subset L .
$$

In fact, since $e *(x * e)=x$, we see that

$$
M=e * L=\bar{L}:=\{\bar{x}: x \in L\} .
$$

From (19) and (20), we see that $L$ (respectively $\bar{L}$ ) is a right (respectively left) ideal of the maximal order $R_{E}$ of $V_{E}$. By Prop. 5.1 (iii), we have

$$
L=\bar{L}=\pi_{E}^{n} R_{E}
$$

for some $n$. But a lattice $L$ of the form $\pi_{E}^{n} R_{E}$ does not satisfy $L=\pi_{E} L^{*}$. Hence, $L$ must be self-dual, in which case $n=0$ and $L=R_{E}$. Hence, $g_{2} \alpha_{2}$ and $g_{3} \alpha_{3}$ are hyperspecial vertices which give rise to the self-dual lattice $R_{E}$. We have thus shown that $y_{0}=g \cdot y$ with $g \in H^{\prime}(E)$ and establish the description of $\mathcal{B}(\operatorname{Spin}(V))$ given in the theorem. Since we have already noted the $S_{3}$-action on $\mathcal{B}(\operatorname{Spin}(V))$, the theorem is proved completely.

Corollary 11.4. - The building $\mathcal{B}(G)$ is the subset of $\mathcal{B}(\operatorname{Spin}(V))$ fixed pointwise by $S_{3}$.

Proof. - We have already seen that $\mathcal{B}(G)$ is the set of self-dual algebra norms. By Cor. 7.5, an element of $\mathcal{B}(G)$ satisfies $\bar{\alpha}=\alpha$ and thus $(\alpha, \alpha, \alpha)$ belongs to $\mathcal{B}(\operatorname{Spin}(V))^{S_{3}}$. Conversely, if $\left(\alpha_{1}, \alpha_{2}, \alpha_{3}\right) \in \mathcal{B}(\operatorname{Spin}(V))^{S_{3}}$, then $\alpha_{1}=\alpha_{2}=\alpha_{3}$ and $\overline{\alpha_{i}}=\alpha_{i}$. This implies that $\alpha_{1}=\alpha_{2}=\alpha_{3}$ is a self-dual norm of octonion algebra and thus $\left(\alpha_{1}, \alpha_{2}, \alpha_{3}\right) \in \mathcal{B}(G)$.

Since $G=\operatorname{Spin}(V)^{\mathbb{Z} / 3 \mathbb{Z}}$, it is natural to ask if $\mathcal{B}(G)=\mathcal{B}(\operatorname{Spin}(V))^{\mathbb{Z} / 3 \mathbb{Z}}$. For some reason, we are unable to deduce this from what we have above, though it must be the case when $p \neq 3$ by the main result of [13].

Let $x$ be a hyperspecial point on $\mathcal{B}(G)$. Then $y=\widetilde{\iota}_{*}(x)$ is also hyperspecial. The link $L(x)$ of $x$ in $\mathcal{B}(G)$ (respectively the link $L(y)$ of $y$ in $\mathcal{B}(\operatorname{Spin}(V))$ is the spherical building of $\underline{G}_{x} \otimes A / \pi$ (respectively $\underline{\operatorname{Spin}(V)} \underline{ }_{y} \otimes A / \pi$ ).

Corollary 11.5. - $L(y)^{S_{3}}=L(x)$.

When $p \neq 2,3$, this corollary also follows from a general result in [13].

TOME $131-2003-\mathrm{N}^{\mathrm{O}} 3$ 


\section{The Anisotropic Case}

We now give some remarks about the non-split case. Let $G$ be an absolute simple algebraic group of type $G_{2}$ over $k$ such that $G$ is non-split. This does not exist if $k$ is a local field. In any case, it is well-known [16] that $G$ can be be realized as the automorphism group of an octonion algebra $V$ over $k$. Therefore, we again have embeddings $G \subset \operatorname{SO}(V, q), G \subset \operatorname{Spin}(V, q)$, where $q$ is the norm form of $V$. There is also an action of $\mathbb{Z} / 3 \mathbb{Z}$ on $\operatorname{Spin}(V, q)$ whose fixed point is precisely $G$.

It is also well-known (see [16]) that in this case, $G, \operatorname{SO}(V, q)$, and $\operatorname{Spin}(V, q)$ are anisotropic. Therefore, $\mathcal{B}(G), \mathcal{B}(\operatorname{SO}(V, q))$ and $\mathcal{B}(\operatorname{Spin}(V, q))$ are singletons. The statement

$$
\mathcal{B}(G)=\mathcal{B}(\operatorname{Spin}(V))^{\mathbb{Z} / 3 \mathbb{Z}}
$$

is therefore trivially true. Moreover, the unique maximinorante norm on $V$ is the norm

$$
\alpha_{0}=\frac{1}{2} \text { ord } \circ q,
$$

and since $V$ is a composition algebra, it follows immediately that $\alpha_{0}$ is an algebra norm. Hence, $\mathcal{B}(G)$ can again be characterized as the subset of maximinorante algebra norms in $\mathcal{B}(\mathrm{SO}(V, q))$. What is not yet apparent is the fact that the map $\iota_{*}: \mathcal{B}(G) \rightarrow \mathcal{B}(\mathrm{SO}(V, q))$ of singleton sets extends to a strong descent datum $\left\{\iota_{E *}\right\}$.

As we have mentioned in the introduction, the result of [6] implies that $G$ has an unramified splitting field $E$. Let $\iota_{E *}: \mathcal{B}\left(G_{E}\right) \rightarrow \mathcal{B}\left(\mathrm{SO}\left(V_{E}, q\right)\right)$ be the unique strong descent map of the buildings of split groups over $E$. By uniqueness, $\iota_{E *}$ is equivariant with respect to the action of $\Gamma=\operatorname{Gal}(E / F)$. Thus, as in the proof of Prop. 4.1, the canonical embedding $\mathcal{B}\left(G_{E}\right) \subset \mathcal{B}\left(\mathrm{SO}\left(V_{E}, q\right)\right)$ induces an embedding $\mathcal{B}\left(G_{E}\right)^{\Gamma}=\mathcal{B}(G) \subset \mathcal{B}\left(\operatorname{SO}\left(V_{E}, q\right)\right)^{\Gamma}=\mathcal{B}(\mathrm{SO}(V, q))$, i.e. $\iota_{*}$ extends to a strong descent datum.

\section{Appendix: Symmetric Spaces of Real Reductive Groups}

In this appendix, we first review the theory of the symmetric space of a real reductive group $G$, manifesting the analogy with the theory of the Bruhat-Tits building in the $p$-adic case. This analogy is also stressed in Springer's article [15]. Here we further emphasize the functoriality of the symmetric spaces, which is better formulated in terms of the notion of extended symmetric space $\mathcal{S}(G)$ introduced below.

Moreover, we show (Thm. 13.11) that if $H$ is a connected real reductive group and $F \subset \operatorname{Aut}(H)$ is a finite group such that $G=\left(H^{F}\right)^{0}$ is also a connected real reductive group, then $\mathcal{S}(H)^{F}=\mathcal{S}(G)$. This leads quickly to a description of the symmetric spaces associated to all classical groups, which again has a $p$ adic counterpart due to Bruhat and Tits. Although this should be well-known, 
we are unable to locate a reference. This formalism also gives immediately $\mathcal{S}(\operatorname{Spin}(8))^{S_{3}}=\mathcal{S}(\operatorname{Spin}(8))^{\mathbb{Z} / 3 \mathbb{Z}}=\mathcal{S}\left(G_{2}\right)$, which is the real version of the main result of $\S 11$ (Cor. 11.4). The $p$-adic analogue of Thm. 13.11 is the main result of $[13]$.

The symmetric space and the extended symmetric space. - Let $H$ be a Lie group in the class considered in [15]. Let $\mathcal{S}_{\text {red }}(H)$ be the set of Cartan involutions on $\mathfrak{h}=$ Lie $H$. Then $\mathcal{S}_{\text {red }}(H)$ is called the (reduced) symmetric space of $H$. It is well-known that a Cartan involution $\theta \in \mathcal{S}_{\text {red }}(H)$ determines a Cartan involution of $H$, and a maximal compact subgroup $K_{\theta}=H^{\theta}$ of $H$. This allows us to identify $\mathcal{S}_{\text {red }}(H)$ with the set of Cartan involutions on $H$, and with the set of maximal compact subgroups of $H$. It is also well-known that $H$ acts transitively on $\mathcal{S}_{\text {red }}(H)$. It is easy to show that the stabilizer of $\theta \in \mathcal{S}_{\text {red }}(H)$ is precisely $K_{\theta}$ when the center $C_{H^{0}}$ of the identity component $H^{0}$ of $H$ is compact.

Let $V=V(H)$ be the maximal vector subgroup of the center $C_{H^{0}}$ of $H^{0}$, and let ${ }^{0} H$ be the subgroup generated by $K_{\theta}$ and the derived group $\left[H^{0}, H^{0}\right]$ of $H^{0}$. We then have

$$
H=V \cdot{ }^{0} H \text {. }
$$

It follows that ${ }^{0} H$ does not depend on the choice of $K_{\theta}$, and the decomposition (21) is a direct product decomposition.

We regard $V=H /{ }^{0} H$ as a homogeneous space of $H$ and define the extended symmetric space $\mathcal{S}(H)$ as the $H$-set $\mathcal{S}_{\text {red }}(H) \times V$.

It is clear that the center of the identity component of ${ }^{0} H$ is compact. It follows that $H$ acts transitively on $\mathcal{S}(H)$ and the stabilizer of $(\theta, v) \in \mathcal{S}(H)$ is precisely $K_{\theta}$. It also follows that the normalizer of $K_{\theta}$ in $H$ is $V . K_{\theta}$.

From now on, we assume that $G$ is a connected reductive group over $\mathbb{R}$. Then $H=G(\mathbb{R})$ is in the class considered in $[15]$. We apply the above to $H$ and define $\mathcal{S}_{\text {red }}(G)=\mathcal{S}_{\text {red }}(G(\mathbb{R})), V(G)=V(G(\mathbb{R})), \mathcal{S}(G)=\mathcal{S}(G(\mathbb{R}))$.

Clearly, $\operatorname{Aut}_{\mathbb{R}}(G)$ acts on $\mathcal{S}_{\text {red }}(G), V(G)$, and $\mathcal{S}(G)$ canonically. We will denote by $G_{x}$ the stabilizer of $x \in \mathcal{S}(G)$ under the action of $G(\mathbb{R})$.

In the sequel, we will make frequent use the following result [14]:

THEOREM 13.1. - Let $G \subset H$ be connected reductive groups over $\mathbb{R}$. Then

(i) If $\theta^{\prime} \in \mathcal{S}_{\text {red }}(H)$ stabilizes $G(\mathbb{R})$, then $\theta^{\prime} \mid G(\mathbb{R}) \in \mathcal{S}_{\text {red }}(G)$.

(ii) For any $\theta \in \mathcal{S}_{\text {red }}(G)$, the set

$$
\left\{\theta^{\prime} \in \mathcal{S}_{\text {red }}(H): \theta^{\prime} \mid G(\mathbb{R})=\theta\right\}
$$

is non-empty, and is permuted transitively by the real points of $Z_{H}(G)$, the centralizer of $G$ in $H$. 
ExAmPLE 13.2. - Let $G=\mathbb{G}_{\mathrm{m}}^{d}$ be an $m$-dimensional split torus. Then $\mathcal{S}_{\text {red }}(G)$ consists of one element, which is $X \mapsto-X$ on Lie $G$ and $g \mapsto g^{-1}$ on $G(\mathbb{R})$. On the other hand, $\mathcal{S}(G)=\left(\mathbb{R}_{>0}^{\times}\right)^{m} \simeq\left(\mathbb{R}^{\times} /\{ \pm 1\}\right)^{m}$.

If $G$ is anisotropic, then $G(\mathbb{R})$ is compact and $\mathcal{S}_{\text {red }}(G)$ consists of only one point, which is $X \mapsto X$ on Lie $G$ and $g \mapsto g$ on $G(\mathbb{R})$.

More generally, if $G$ is anisotropic modulo its center, then $\mathcal{S}_{\text {red }}(G)$ consists of one point only.

REMARK 13.3. - The class considered by [15] contains some disconnected groups. However, it does not contain the group $H=G(\mathbb{R})$, where $G$ is the full orthogonal group of a quadratic form in two variables.

Apartments. - For each maximal $\mathbb{R}$-split torus $S$ of $G$, let $\mathcal{A}_{\text {red }}(S)=\{\theta \in$ $\left.\mathcal{S}_{\text {red }}(G): \theta(S(\mathbb{R})) \subset S(\mathbb{R})\right\}$. We call $\mathcal{A}_{\text {red }}(S)$ the apartment of $S$. We notice that $\theta \in \mathcal{A}_{\text {red }}(S)$ if and only if $\theta$ extends the only Cartan involution of $S(\mathbb{R})$.

Let $Z=Z_{G}(S)$. If $\theta \in \mathcal{A}_{\text {red }}(S)$, and $z \in G(\mathbb{R})$ commutes with $S(\mathbb{R})$, then $\theta(z)$ commutes with $\theta(S(\mathbb{R}))=S(\mathbb{R})$. Therefore, $\theta$ stabilizes $Z(\mathbb{R})$ and hence defines a Cartan involution on $Z(\mathbb{R})$ by Thm. 13.1 (i). Since $Z$ is anisotropic modulo its center, $\theta$ extends the only Cartan involution of $Z(\mathbb{R})$.

- All apartments are conjugate. This is obvious.

- Each point lies in an apartment. The unique Cartan involution of $S(\mathbb{R})$ always extends to a Cartan involution of $G(\mathbb{R})$ by Thm. 13.1 (ii). Therefore, at least one Cartan involution lies on an apartment. It follows that any Cartan involution lies on an apartment.

- $S(\mathbb{R})$ acts on $\mathcal{A}_{\text {red }}(S)$ transitively. If $\theta_{1}$ and $\theta_{2}$ both extend the only Cartan involution of $S$, then $\theta_{2}=z . \theta_{1}$ are conjugate by some $z$ of $Z(\mathbb{R})$ by Thm. 13.1 (ii). We can write $z=s k$, where $z \in S(\mathbb{R})$ and $k$ is in the maximal compact subgroup of $Z(\mathbb{R})$ (Cartan decomposition). Since $k \in K_{\theta_{1}}$, we have $K_{\theta_{2}}=z K_{\theta_{1}} z^{-1}=s K_{\theta_{1}} s^{-1}$. Hence $\theta_{2}=s . \theta_{1}$.

- The dimension of $\mathcal{A}_{\text {red }}(S)$ is $\operatorname{rank}_{\mathbb{R}} G-\operatorname{rank}_{\mathbb{R}} Z(G)$. Let $\theta \in \mathcal{A}_{\text {red }}(S)$. The normalizer of $K_{\theta}$ in $G(\mathbb{R})$ is $C_{G}(\mathbb{R}) \cdot K_{\theta}$, where $C_{G}$ is the center of $G$. In particular, the stabilizer of $\theta$ in $S(\mathbb{R})$ is $S(\mathbb{R}) \cap C_{G}(\mathbb{R}) \cdot K_{\theta}$.

- Any two points $x, y \in \mathcal{S}_{\text {red }}(G)$ lie on some apartment $\mathcal{A}_{\text {red }}(S)$. Suppose that $x \in \mathcal{A}_{\text {red }}(S)$. Then we have the Cartan decomposition $G(\mathbb{R})=K_{x} S(\mathbb{R}) K_{x}$. Suppose that $K_{y}=g K_{x} g^{-1}$ with $g=k s k^{\prime}$, where $k, k^{\prime} \in K_{x}$ and $s \in S$. Then it is clear that $K_{y}=(k s) K_{x}(k s)^{-1}$ and $y$ lies on the apartment associated to $(k s) S(k s)^{-1}=k S k^{-1}$. It is also clear that $x$ lies in $k S k^{-1}$ also since $\theta_{x}\left(k S k^{-1}\right)=\theta_{x}(k) \theta_{x}(S) \theta_{x}(k)^{-1}=k S k^{-1}$. Thus $x, y$ both lie on $\mathcal{A}_{\text {red }}\left(k S k^{-1}\right)$.

- $K_{x}$ permutes apartments containing $x$ transitively. Suppose that $x \in$ $\mathcal{A}_{\text {red }}(S)$ and $x \in \mathcal{A}_{\text {red }}\left(S^{\prime}\right)$. We may assume that $S^{\prime}=g S g^{-1}$. The points of $\mathcal{S}_{\text {red }}(G)$ on $\mathcal{A}_{\text {red }}\left(S^{\prime}\right)$ are precisely $g S(\mathbb{R}) g^{-1} \cdot(g x)=g S(\mathbb{R}) \cdot x$. Therefore, there exists $s \in S(\mathbb{R})$ such that $x=g s \cdot x$. Let $k=g s$, then we have $k \in K_{x}$ and $k S k^{-1}=S^{\prime}$. Therefore, $\mathcal{A}_{\text {red }}\left(S^{\prime}\right)=k \cdot \mathcal{A}_{\text {red }}(S)$. 
- Another description of the apartments containing $x$. Let $\mathfrak{g}=\mathfrak{k} \oplus \mathfrak{p}$ be the Cartan decomposition relative to $\theta_{x}$. Then there is a bijection between the apartments containing $x$ and the maximal abelian sub-algebras of $\mathfrak{p}$. The bijection is $\mathcal{A}_{\text {red }}(S) \mapsto$ Lie $S$. Then the previous statement is the well-known fact that $K_{x}$ permutes the abelian sub-algebras of $\mathfrak{p}$.

We also define the extended apartment $\mathcal{A}(S)$ as $\mathcal{A}_{\text {red }}(S) \times V(G)$. In particular, $\operatorname{dim} \mathcal{A}(S)=\operatorname{dim} S$.

Functoriality. - Assume that $G, H$ are reductive groups over $\mathbb{R}$ such that $G \subset H$. Suppose that $x \in \mathcal{S}(G), y \in \mathcal{S}(H)$ are such that the associated maximal compact subgroups $G_{x} \subset G(\mathbb{R}), H_{y} \subset H(\mathbb{R})$ satisfy $G_{x} \subset H_{y}$, then we have a $G(\mathbb{R})$-equivariant map $\mathcal{S}(G) \rightarrow \mathcal{S}(H)$, sending $g \cdot x$ to $g \cdot y$. It is easy to see that $H_{y} \cap G(\mathbb{R})=G_{x}$, hence this equivariant map is always injective.

However, the Cartan involution $\theta_{y}$ associated to $y$ may not stabilize $G$.

ExAmPLE 13.4. - Let $G=\mathrm{SL}_{2}, H=\mathrm{SL}_{4}$, and define the inclusion $\iota: G \hookrightarrow H$ by $g \mapsto\left(\begin{array}{cc}g & 0 \\ 0 & { }^{t} g^{-1}\end{array}\right)$. The standard maximal compact subgroup $K$ of $G(\mathbb{R})$ is the stabilizer of the quadratic form represented by the $2 \times 2$ identity matrix $I_{2}$.

It is easy to see that $\iota K$ stabilizes the quadratic form $q$ represented by the $4 \times 4$ matrix $\left(\begin{array}{ll}a I_{2} & b I_{2} \\ b I_{2} & c I_{2}\end{array}\right)$. When $q$ is positive definite (for example, $a=c=1$ and $b$ sufficiently small), the stabilizer $K_{q}$ of $Q_{q}$ in $H(\mathbb{R})$ is a maximal compact subgroup such that $\iota K \subset K_{q}$. But the Cartan involution $\theta_{q}$ associated to $K_{q}$ does not stabilize $\iota G(\mathbb{R})$ as long as $b \neq 0$.

Definition 13.5. - (i) We say that the map $\mathcal{S}(G) \rightarrow \mathcal{S}(H), g \cdot x \mapsto g \cdot y$ is a descent map if $\theta_{y}$ stabilizes $G(\mathbb{R})$ (and hence $\theta_{y}$ descends to $\theta_{x}:\left.\theta_{y}\right|_{G(\mathbb{R})}=\theta_{x}$ ).

(ii) We say that $\mathcal{S}(G) \rightarrow \mathcal{S}(H)$ is a toral map if there exists a maximal $\mathbb{R}$-split torus $S$ of $G$, a maximal $\mathbb{R}$-split torus $T$ of $G$ such that $x \in \mathcal{A}(S), y \in \mathcal{A}(T)$, and $S \subset T$.

Proposition 13.6. - (i) A descent map is a toral map.

(ii) A toral map is a descent map.

(iii) If $\mathcal{S}(G) \rightarrow \mathcal{S}(H)$ is a descent map then $\theta_{y^{\prime}} \mid G(\mathbb{R})=\theta_{x^{\prime}}$ for all $x^{\prime} \in \mathcal{S}(G)$, where $y^{\prime}$ is the image of $x^{\prime}$.

Proof. — Let $\mathfrak{g}=\mathfrak{k} \oplus \mathfrak{p}$ and $\mathfrak{h}=\mathfrak{k}_{H} \oplus \mathfrak{p}_{H}$ be the Cartan decomposition associated to $\theta_{x}$ and $\theta_{y}$ respectively.

(i) Choose $S \subset G$ such that $x \in \mathcal{A}(S)$. Then $\mathfrak{s}=$ Lie $S$ is an abelian subalgebra of $\mathfrak{p} \subset \mathfrak{p}_{H}$. Let $\mathfrak{t}$ be a maximal abelian sub-algebra of $\mathfrak{p}_{H}$ containing $\mathfrak{s}$. Then $\mathfrak{t}=\operatorname{Lie} T$ for some maximal $\mathbb{R}$-split torus $T$ of $H$. By construction, we have $S \subset T$ and $y \in \mathcal{A}(T)$.

(ii) We need to show that $\mathfrak{k} \subset \mathfrak{k}_{H}$ and $\mathfrak{p} \subset \mathfrak{p}_{H}$.

It is clear that $\mathfrak{k}=\operatorname{Lie} G_{x}$ is contained in $\mathfrak{k}_{H}=$ Lie $H_{y}$. By our assumption, there is a maximal abelian sub-algebra $\mathfrak{a}$ of $\mathfrak{p}$, and there is a maximal abelian

TOME $131-2003-\mathrm{N}^{\mathrm{O}} 3$ 
sub-algebra $\mathfrak{a}_{H}$ of $\mathfrak{p}_{H}$ such that $\mathfrak{a} \subset \mathfrak{a}_{H}$. Since $\mathfrak{p}=\bigcup_{k \in G_{x}} k \cdot \mathfrak{a}$, it follows immediately that $\mathfrak{p} \subset \mathfrak{p}_{H}$. Now (ii) is proved.

(iii) is obvious.

Base change. - Denote $\operatorname{Res}_{\mathbb{C} / \mathbb{R}}(G \otimes \mathbb{C})$ by $G_{\mathbb{C}}$. Then there is a canonical map $\mathcal{S}_{\text {red }}(G) \rightarrow \mathcal{S}_{\text {red }}\left(G_{\mathbb{C}}\right)$. It is constructed as follows: let $\theta \in \mathcal{S}_{\text {red }}(G)$, and let $\mathfrak{k}$ and $\mathfrak{p}$ be the +1 and -1 eigenspaces of $\theta$ on $\mathfrak{g}=\operatorname{Lie} G$. Then a Cartan involution $\theta_{\mathbb{C}}$ on $\mathfrak{g}_{\mathbb{C}}=\mathfrak{g} \otimes \mathbb{C}=\operatorname{Lie} G_{\mathbb{C}}$ is defined by $\theta_{\mathbb{C}}(k+p)=\bar{k}-\bar{p}$ for all $k \in \mathfrak{k} \otimes \mathbb{C}, p \in \mathfrak{p} \otimes \mathbb{C}$.

Now the base change map $\mathcal{S}_{\text {red }}(G) \rightarrow \mathcal{S}_{\text {red }}\left(G_{\mathbb{C}}\right)$ is $\theta \mapsto \theta_{\mathbb{C}}$. It is easy to see that this map is $G(\mathbb{R})$-equivariant.

We also have an obvious inclusion $V(G) \hookrightarrow V\left(G_{\mathbb{C}}\right)$. Combining with the previous construction, we get a base change map

$$
\mathcal{S}(G) \longrightarrow \mathcal{S}\left(G_{\mathbb{C}}\right)
$$

which is clearly a $G(\mathbb{R})$-equivariant descent map.

Proposition 13.7. - The image of the base change descent map $\mathcal{S}(G) \rightarrow$ $\mathcal{S}\left(G_{\mathbb{C}}\right)$ is $\mathcal{S}\left(G_{\mathbb{C}}\right)^{\mathrm{Gal}(\mathbb{C} / \mathbb{R})}$.

Proof. - This can be checked directly. It also follows immediately from the proof of Thm. 13.11.

Proposition 13.8. - Assume that $G \subset H$ is an embedding of complex groups in the sense that there are complex reductive groups $G^{\prime} \subset H^{\prime}$ such that $G=$ $\operatorname{Res}_{\mathbb{C} / \mathbb{R}} G^{\prime}$ and $H=\operatorname{Res}_{\mathbb{C} / \mathbb{R}} H^{\prime}$. Then any equivariant map $\mathcal{S}(G) \rightarrow \mathcal{S}(H)$ determined by an inclusion $G_{x} \subset H_{y}$ is a descent map.

Proof. - In this case, $\mathfrak{g}$ can be identified with the complex Lie algebra $\mathfrak{g}^{\prime}=$ Lie $G^{\prime}$ with the complex structure forgotten.

Then the Cartan involution $\theta_{x}$ on $\mathfrak{g}$ is determined by $K_{x}$ as follows: $\theta_{x}(X)=X$ on $\mathfrak{k}=\operatorname{Lie} G_{x}$, and $\theta_{x}(X)=-X$ for $X \in i \mathfrak{k}$. This describes $\theta_{x}$ on $\mathfrak{g}$ completely since $\mathfrak{g}=\mathfrak{k}+i \mathfrak{k}$.

The same description applies to $\theta_{y}$ as well. It follows immediately that $\theta_{y} \mid \mathfrak{g}=\theta_{x}$.

Descent maps and base change. - Let $\iota: G \subset H$ be an inclusion of real reductive groups. Assume $x \in \mathcal{S}(G), y \in \mathcal{S}(H)$ are such that $G_{x} \subset H_{y}$ and denote by $\iota_{*}$ the equivariant map $\mathcal{S}(G) \rightarrow \mathcal{S}(H), g \cdot x \mapsto g \cdot y$.

Proposition 13.9. - The map $\iota_{*}$ is a descent map if and only if $\iota_{*}$ extends to a $G_{\mathbb{C}}(\mathbb{R})$-equivariant map $\mathcal{S}\left(G_{\mathbb{C}}\right) \rightarrow \mathcal{S}\left(H_{\mathbb{C}}\right)$.

Proof. - The "only if" part is clear from the description of base change. The "if" part follows from Prop. 13.8. 
REMARK 13.10. - We may regard $\theta_{x}$ as the counterpart of the valuation of root datum in the $p$-adic case. The real case is a lot easier than the $p$-adic case because $\theta_{x}$ is really defined on $G(\mathbb{R})$ and can be restricted on any subgroup directly. In the case of valuation of root datum, the valuation is only defined on various root subgroups, and to talk about descent, one needs to start in a situation where the toral condition is satisfied.

Prop. 13.6 is essentially [14, Chap. II, Thm. 2.4]. Its $p$-adic analogue is shown in [18]. Prop. 13.8 is an analogue of Landvogt's criterion for toral map [11, 2.4.1]. Prop. 13.9 says that in the real case, there is no need for the notion of a strong descent map. The existence of descent maps for arbitrary $G \subset H$ is the main result of [11], the corresponding real analogue is an easy consequence of Thm. 13.1.

Finite group actions. - Suppose that we have a finite group $F$ acting on $H$. Then $G=\left(H^{F}\right)^{0}$ is also reductive (see, for example, [13]). We denote the inclusion $G \rightarrow H$ by $\iota$.

THEOREM 13.11. - Let $X$ be the set of descent maps $\iota_{*}: \mathcal{S}(G) \rightarrow \mathcal{S}(H)$ satisfying $\iota_{*}(\mathcal{S}(G)) \subset \mathcal{S}(H)^{F}$. Then,

(i) $X$ is non-empty.

(ii) $\iota_{*}(\mathcal{S}(G))=\mathcal{S}(H)^{F}$ for all $\iota_{*} \in X$.

(iii) $X$ is a principal homogeneous space of $V(G)$.

Proof. - We will first assume (i) and prove (ii) and (iii).

(ii) Let $\iota_{*} \in X$. Choose $x \in \mathcal{S}(G)$ and let $y=\iota_{*}(x)$. Let $\mathfrak{h}=\mathfrak{k}_{H}+\mathfrak{p}_{H}$ and $H(\mathbb{R})=H_{y} \cdot P$ be the Cartan decomposition of $\mathfrak{h}$ and $H(\mathbb{R})$ associated to $\theta_{y}$, where $P=\exp \left(\mathfrak{p}_{H}\right)$. Then every point $y^{\prime}$ on $\mathcal{S}(H)$ can be expressed as $p . y$ for a unique $p \in P$. We notice that $\mathfrak{k}_{H}, \mathfrak{p}_{H}$ and $H_{y}$ are $F$-stable since $y$ is fixed by $F$. Since the isomorphism exp $: \mathfrak{p}_{H} \rightarrow P$ is $F$-equivariant, $P$ is also $F$-stable.

Assume that $y^{\prime}=p . y$ is fixed by $F$. Then for each $s \in F$, we have $y^{\prime}=$ $s\left(y^{\prime}\right)=s(p) \cdot s(y)=s(p) \cdot y$. Since $s(p) \in P$, we must have $s(p)=p$. Now observe that $P^{F}=\exp \left(\mathfrak{p}_{H}{ }^{F}\right)$ is connected. This implies that $p \in G(\mathbb{R}) \subset\left(H(\mathbb{R})^{F}\right)^{0}$. Thus $y^{\prime}=\iota_{*}(p \cdot x)$ is in the image of $\iota_{*}$.

(iii) It is clear that $V(G)$ acts on $X$ by $\left(v \cdot \iota_{*}\right)(x)=\iota_{*}\left(v^{-1} \cdot x\right)$. Let $\iota_{*}, \iota_{*}^{\prime}$ be two elements of $X$. Choose $y \in \mathcal{S}(H)^{F}$ and let $x=\iota_{*}^{-1}(y), x^{\prime}=\left(\iota_{*}^{\prime}\right)^{-1}(y)$. Then the restriction of $\theta_{y}$ on $G(\mathbb{R})$ is equal to both $\theta_{x}$ and $\theta_{x^{\prime}}$. It follows that $x^{\prime}=v \cdot x$ for some $v \in V(G)$. Thus we have shown that $V(G)$ acts transitively on $X$. It is clear that the stabilizer of any point $\iota_{*} \in X$ is trivial. Therefore, $X$ is a principal homogeneous space of $V(G)$.

We now prove (i) when both $G$ and $H$ are complex groups in the sense of Prop. 13.8. Choose a point $x$ on $\mathcal{S}(G)$. Recall that $F \ltimes H(\mathbb{R})$ acts on $\mathcal{S}(H)$ by isometries. In particular, the compact group $F \times G_{x}$ acts on $\mathcal{S}(H)$. By the

TOME $131-2003-\mathrm{N}^{\mathrm{O}} 3$ 
fixed point lemma $[15,5.28], F \times G_{x}$ fixes a point $y$ on $\mathcal{S}(H)$. By Prop. 13.8, the map $\iota_{*}: g \cdot x \mapsto g \cdot y$ is an element of $X$.

Finally, we can now prove (i) in general. Let $X_{\mathbb{C}}$ be the set of descent maps $\iota_{\mathbb{C}}: \mathcal{S}\left(G_{\mathbb{C}}\right) \rightarrow \mathcal{S}\left(H_{\mathbb{C}}\right)$ such that $\iota_{\mathbb{C}}\left(\mathcal{S}\left(G_{\mathbb{C}}\right)\right) \subset \mathcal{S}\left(H_{\mathbb{C}}\right)^{F}$. We have shown that $X_{\mathbb{C}}$ is non-empty and is an affine space under $V\left(G_{\mathbb{C}}\right)$. It is easy to see that $\Gamma=\operatorname{Gal}(\mathbb{C} / \mathbb{R})$ acts on $X_{\mathbb{C}}$ by affine transformation. Therefore, we can find $\iota_{\mathbb{C}} \in X_{\mathbb{C}}$ which is $\Gamma$-equivariant. It follows that $\iota_{\mathbb{C}}$ restricts to a map from $\mathcal{S}\left(G_{\mathbb{C}}\right)^{\Gamma}=\mathcal{S}(G)$ to $\mathcal{S}\left(H_{\mathbb{C}}\right)^{\Gamma}=\mathcal{S}(H)$ by Prop. 13.7. This restriction $\iota_{*}=\iota_{\mathbb{C}} \mid \mathcal{S}(G)$ is then a descent map $\mathcal{S}(G) \rightarrow \mathcal{S}(H)$ by Prop. 13.9. It is clear that $\iota_{*} \in X$ and hence (i) is proved for arbitrary $G$ and $H$.

Symmetric space as a space of norms. - in the rest of this section we give some examples to illustrate Thm.13.11. Let $D$ be $\mathbb{R}, \mathbb{C}$, or $\mathbb{H}$. Let $V$ be a vector space over $D$. By a hermitian norm (or simply a norm) on $V$ we mean a function $\alpha: V \rightarrow \mathbb{R}$ satisfying the following condition: there exists a (unique) positive definite hermitian form $\langle-,-\rangle=\langle-,-\rangle_{\alpha}$ on $V$ such that $\alpha(v)=\langle v, v\rangle^{1 / 2}$.

Let $G=\mathrm{GL}_{D}(V)$. It is well-known that $\mathcal{S}(G)$ can be identified with the set $\mathcal{N}(V)$ of hermitian norms $\alpha$ on $V$

For a general connected reductive group, choose an embedding $f: G \rightarrow$ $\mathrm{GL}_{D}(V)$ and a descent map $f_{*}: \mathcal{S}(G) \rightarrow \mathcal{S}\left(\mathrm{GL}_{D}(V)\right)$ (whose existence follows from Thm. 13.1). Then we can identify $\mathcal{S}(G)$ with the image of $f_{*}$, hence obtain a model of $\mathcal{S}(G)$ as a set of hermitian norms.

As an example, consider the isomorphism $f: \mathrm{GL}_{H}(V) \rightarrow \mathrm{GL}_{H}\left(V^{*}\right)$. This gives us a bijection from $\mathcal{S}(V)=\mathcal{N}(V)$ to $\mathcal{S}(V)=\mathcal{N}\left(V^{*}\right)$. In other words, a hermitian norm $\alpha$ on $V$ determines a unique hermitian form $\alpha^{*}$ on $V^{*}$, and conversely.

Let $\epsilon \in\{ \pm 1\}$, let $V$ be a vector space over $D$, and let $\langle-,-\rangle$ be a nondegenerate $\epsilon$-hermitian form on $V$. Then $G=\operatorname{Aut}_{D}(V,\langle-,-\rangle)^{0}$ is a classical group, and is the identity component of the fixed points of the involution $\iota$ : $g \mapsto g^{*}$ on $\mathrm{GL}_{D}(V)$, where $g^{*}$ is defined by $\left\langle g v, g^{*} w\right\rangle=\langle v, w\rangle$ for all $v, w \in V$.

The involution $\iota$ acts on $\mathcal{S}\left(\mathrm{GL}_{D}(V)\right)=\mathcal{N}(V)$. It is easy to see that $\iota \alpha=\alpha$ if and only if $\alpha=\alpha^{*}$ when we identify $V$ with $V^{*}$ via $\langle-,-\rangle$. We call such a norm a self-dual norm. By Thm.13.11, we may identify $\mathcal{S}(G)$ with the set of self-dual norms on $V$.

Let $F$ be $\mathbb{R}$ or $\mathbb{C}$ and let $V$ be an octonion algebra over $F$. Then $V$ is equipped with a natural quadratic form $q$. Let $G$ be the automorphism group of the octonion algebra $V$ (as an algebraic group over $\mathbb{R}$ ). Then $G$ is of type $G_{2}$ and there are natural embeddings $G \hookrightarrow \operatorname{SO}(V, q)$ and $G \hookrightarrow \operatorname{Spin}(V, q)$.

It is easy to see that the centralizer of $G$ in $\operatorname{SO}(V, q)$ (resp. in $\operatorname{Spin}(V, q)$ ) is the center of $\mathrm{SO}(V, q)$ (resp. of $\operatorname{Spin}(V, q)$ ). By Thm. 13.1, there is a unique descent map $\mathcal{S}(G) \hookrightarrow \mathcal{S}(\mathrm{SO}(V, q))=\mathcal{S}(\operatorname{Spin}(V, q))$. 
As in $\S 11$, there is a natural action of $\mathbb{Z} / 3 \mathbb{Z}$ on $\operatorname{Spin}(V, q)$ such that $G=$ $\operatorname{Spin}(V, q)^{\mathbb{Z} / 3 \mathbb{Z}}$. By Thm. 13.11, the image of $\mathcal{S}(G)$ in $\mathcal{S}(\operatorname{Spin}(V, q))$ is precisely $\mathcal{S}(\operatorname{Spin}(V, q))^{\mathbb{Z} / 3 \mathbb{Z}}$.

\section{Appendix: The Symmetric Space of $G_{2}(\mathbb{R})$ and $G_{2}(\mathbb{C})$}

In this appendix, we will show a real version of Thm. 7.2 , i.e. that $\mathcal{S}\left(G_{2}\right)$ is the space of self-dual algebra hermitian norms.

Definition 14.1. - Again $F$ is either $\mathbb{R}$ or $\mathbb{C}$. By a normed octonion algebra over $F$, we mean a pair $(V, \alpha)$ where $V$ is an octonion algebra over $F$, and $\alpha: V \rightarrow \mathbb{R}$ is a hermitian norm on $V$ satisfying the following two conditions

$(\mathrm{AN}): \alpha$ is an algebra norm in the sense that $\alpha(x) \cdot \alpha(y) \geq \alpha(x \cdot y)$ for all $x, y \in V$

(SD): $\langle-,-\rangle_{\alpha}$ is self-dual with respect to the symmetric bilinear form $B(-,-): V \times V \rightarrow F$ defined by $B(x, y)=\operatorname{Tr}(x \cdot \bar{y})$.

An isomorphism between normed octonions $(V, \alpha)$ and $\left(V^{\prime}, \alpha^{\prime}\right)$ is an algebra isomorphism $f: V \rightarrow V^{\prime}$ such that $\alpha^{\prime} \circ f=\alpha$.

Proposition 14.2. - (i) Let $(V, \alpha)$ and $\left(V^{\prime}, \alpha^{\prime}\right)$ be two normed octonion algebras over $F$. Then $(V, \alpha)$ is isomorphic $\left(V^{\prime}, \alpha^{\prime}\right)$ if and only if $V$ is isomorphic to $V^{\prime}$ as algebras over $F$.

(ii) Let $V$ be a fixed octonion algebra over $F$. Let $\mathcal{N}$ be the set of $\alpha: V \rightarrow \mathbb{R}$ such that $(V, \alpha)$ is a normed octonion. Then $\operatorname{Aut}(V)$ acts on $\mathcal{N}$ by $g \cdot \alpha=\alpha \circ g^{-1}$ and the action is transitive.

Proof. - Let $\mathcal{N}^{\prime}$ be the set of hermitian norms $\alpha$ satisfying (SD). Since $B(x, y)$ $=B(g \cdot x, g \cdot y)$ for all $g \in \operatorname{Aut}(V)$, it is clear that $\operatorname{Aut}(V)$ acts on $\mathcal{N}^{\prime}$ by $g \cdot \alpha=\alpha \circ g^{-1}$ and $\mathcal{N}$ is stable under this action.

It is also clear that (i) is equivalent to (ii). Therefore, it suffices to prove the transitivity statement in (ii). We shall first find a nice set representing every orbit in $\operatorname{Aut}(V) \backslash \mathcal{N}^{\prime}$ and then show that there is at most one orbit which may consists of hermitian norms satisfying $(\mathrm{AN})$.

If $F=\mathbb{R}$ and $V$ is non-split, then $B(-,-)$ is positive definite. In this case, $\alpha: x \mapsto\left(\frac{1}{2} B(x, x)\right)^{1 / 2}$ is the only norm which satisfies condition (SD). It is clear that it also satisfies (AN). Therefore, the proposition is trivial in this case.

From now on, we assume that $V$ is a split octonion $(F$ can be $\mathbb{R}$ or $\mathbb{C})$. We may further assume that $V$ is the so-called Zorn's model. The notation here is the same as that in $\S 5$.

It is known (see [10, Cor. 33.21]) that $G_{2}(F)=\operatorname{Aut}(V)$ acts transitively on the set

$$
S=\{v \in V: \operatorname{Tr}(v)=1 \text { and } B(v, v)=0\} .
$$

TOME $131-2003-\mathrm{N}^{\mathrm{O}} 3$ 
Choose $v_{0} \in S$ such that $\alpha\left(v_{0}\right)=\inf \{\alpha(v): v \in S\}$, and then choose $g \in G_{2}(F)$ such that $g \cdot v_{0}=e_{4} \in S$. By replacing $\alpha$ by $\alpha \circ g^{-1}$, we may assume that $\alpha\left(e_{4}\right)=\inf \{\alpha(v): v \in S\}$.

Observe that for $i \in\{-3,-2,-1,1,2,3\}, e_{4}+t e_{i} \in S$ for all $t \in F$. Therefore, $\alpha\left(e_{4}+t e_{i}\right) \geq \alpha\left(e_{4}\right)$. This implies immediately that $e_{4} \perp e_{i}$ with respect to $\langle-,-\rangle_{\alpha}$.

Let $Q=\left(\left\langle e_{i}, e_{j}\right\rangle_{\alpha}\right)$ and $J=\left(B\left(e_{i}, e_{j}\right)\right)$ be the matrices of $\langle-,-\rangle_{\alpha}$ and $B(-,-)$ with respect to the basis $\left(e_{4}, e_{-4}, e_{1}, e_{2}, e_{3}, e_{-1}, e_{-2}, e_{-3}\right)$. Then the condition (SD) is simply that $Q^{t} \bar{J}^{-1} Q=J$. It is easy to see that

$$
J=\left(\begin{array}{cccc}
0 & 1 & 0 & 0 \\
1 & 0 & 0 & 0 \\
0 & 0 & 0 & I_{3} \\
0 & 0 & I_{3} & 0
\end{array}\right)
$$

where $I_{3}$ is the $3 \times 3$ identity matrix. An easy computation shows that we have $e_{-4} \perp e_{i}$ for $i \in\{-3,-2,-1,1,2,3\}$ and $e_{4} \perp e_{-4}$. Therefore, we can write

$$
Q=\left(\begin{array}{cccc}
r & 0 & 0 & 0 \\
0 & s & 0 & 0 \\
0 & 0 & A & \bar{B}^{t} \\
0 & 0 & B & C
\end{array}\right)
$$

The condition (SD) implies

$$
\begin{aligned}
B^{t} \cdot A+A^{t} \cdot B & =0, \\
C^{t} \cdot A+\bar{B} \cdot B & =I, \\
C^{t} \cdot \bar{B}^{t}+\bar{B} \cdot C & =0 .
\end{aligned}
$$

Let $W=F \cdot e_{1}+F \cdot e_{2}+F \cdot e_{3}$. The group $\mathrm{SL}(W) \simeq \mathrm{SL}_{3}(F)$ acts on Zorn's model by

$$
g \cdot\left(\begin{array}{ll}
a & w \\
\varphi & b
\end{array}\right)=\left(\begin{array}{cc}
a & g \cdot w \\
\varphi \circ g^{-1} & b
\end{array}\right)
$$

Therefore, if we replace $\alpha$ with $\alpha \circ g$ for $g \in \operatorname{SL}(W) \hookrightarrow \operatorname{Aut}(V)$, then $Q$ is replace by

$$
\left(\begin{array}{cccc}
r & 0 & 0 & 0 \\
0 & s & 0 & 0 \\
0 & 0 & \bar{g}^{t} \cdot A \cdot g & \bar{g}^{t} \cdot \bar{B}^{t} \cdot\left(g^{t}\right)^{-1} \\
0 & 0 & \bar{g}^{-1} \cdot B \cdot g & \bar{g}^{-1} \cdot C \cdot\left(g^{t}\right)^{-1}
\end{array}\right)
$$

We may thus assume that $A=\operatorname{diag}(1,1, u)$ with $u>0$ by applying a suitable $g$.

The matrix $A=\operatorname{diag}(1,1, u)$ defines a positive definite hermitian form $h_{A}$ on $W \simeq F^{3}$. Let $H=\operatorname{Aut}\left(h_{A}\right) \cap \operatorname{SL}(W)$, so $H$ is isomorphic to $\mathrm{SO}(3)$ when $F=\mathbb{R}$ and to $\mathrm{SU}(3)$ when $F=\mathbb{C}$. If we replace $\alpha$ by $\alpha \circ g$ with $g \in H \hookrightarrow \operatorname{Aut}(V)$, then $A$ is unchanged, and $B$ is replaced by $A^{-1} \cdot g^{t} \cdot A \cdot B \cdot g$.

BULletin DE LA SOCIÉtÉ MATHÉMATIQUE DE FRANCE 
Let $W^{\prime}$ be the space of $3 \times 3$ matrices $B$ over $F$ such that $A \cdot B+B^{t} \cdot A=0$. Then $H$ acts on $W^{\prime}$ by

$$
(g, B) \longmapsto A^{-1} \cdot g^{t} \cdot A \cdot B \cdot g .
$$

It is easy to see that $W^{\prime}$ is isomorphic to $\bigwedge^{2} W$ as an $H$-module, and that every element of $W^{\prime}$ is $H$-conjugate to an element of the form

$$
B=\left(\begin{array}{ccc}
0 & v & 0 \\
-v & 0 & 0 \\
0 & 0 & 0
\end{array}\right)
$$

Therefore, we may assume that the sub-matrix $B$ of $Q$ indeed has the above form by applying a suitable $h$. After these normalizations, a simple computation shows that $Q$ has the following form:

$$
Q=\left(\begin{array}{cccccccc}
r & 0 & 0 & 0 & 0 & 0 & 0 & 0 \\
0 & s & 0 & 0 & 0 & 0 & 0 & 0 \\
0 & 0 & 1 & 0 & 0 & 0 & -\bar{v} & 0 \\
0 & 0 & 0 & 1 & 0 & \bar{v} & 0 & 0 \\
0 & 0 & 0 & 0 & u & 0 & 0 & 0 \\
0 & 0 & 0 & v & 0 & 1+|v|^{2} & 0 & 0 \\
0 & 0 & -v & 0 & 0 & 0 & 1+|v|^{2} & 0 \\
0 & 0 & 0 & 0 & 0 & 0 & 0 & u^{-1}
\end{array}\right) .
$$

We now apply the condition (AN), which has not been used so far. We have $\alpha\left(e_{4}\right)=\sqrt{r}$. Since $\alpha\left(e_{4}\right) \cdot \alpha\left(e_{4}\right) \geq \alpha\left(e_{4} \cdot e_{4}\right)=\alpha\left(e_{4}\right)$, we have $r \geq 1$. Similarly, from $e_{-4} \cdot e_{-4}=e_{-4}$ we deduce that $s \geq 1$. However, the condition $Q^{t} \cdot \bar{J}^{-1} \cdot Q=J$ implies that $r s=1$. Therefore, we have $r=s=1$.

Let $x=\bar{v} e_{1}+e_{-2}$. Then $\alpha(x)=1$ and $x \cdot e_{-3}=-e_{1}$. Therefore, $\alpha(x)$. $\alpha\left(e_{-3}\right) \geq \alpha\left(-e_{1}\right)$. That is, $1 \cdot u^{-1} \geq 1$. So $u \leq 1$.

Since $e_{1} \cdot e_{2}=-e_{-3}$, we have $\alpha\left(e_{1}\right) \cdot \alpha\left(e_{2}\right) \geq \alpha\left(-e_{-3}\right)$. That is, $1 \cdot 1 \geq u^{-1}$. So $u \geq 1$ and hence $u=1$.

Since $e_{2} \cdot e_{3}=-e_{-1}$, we have $1 \cdot 1 \geq 1+|v|^{2}$. Therefore, $v=0$.

We now conclude that $Q$ is the $8 \times 8$ identity matrix and hence there is at most one $\operatorname{Aut}(V)$-orbit in $\mathcal{N}$. The proposition is proved completely.

Proposition 14.3. - For any octonion algebra $V$, there exists $\alpha$ such that $(V, \alpha)$ is a normed octonion algebra.

Proof. — As we have remarked before, the case when $F=\mathbb{R}$ and $V$ is non-split is obvious.

Now let $F$ be either $\mathbb{R}$ or $\mathbb{C}$ and let $V$ again be Zorn's model. It is clear that

$$
\alpha: \sum_{1 \leq|i| \leq 4} a_{i} e_{i} \longmapsto \sqrt{\sum_{1 \leq|i| \leq 4}\left|a_{i}\right|^{2}}
$$

TOME $131-2003-\mathrm{N}^{\mathrm{O}} 3$ 
satisfies (SD). In the preceding proposition, we show that it is the only hermitian norm (up to conjugacy by $\operatorname{Aut}(V)$ ) that has a chance to satisfy both (AN) and (SD). We now show that it indeed satisfies (AN).

In fact, let $x=\sum a_{i} e_{i}, y=\sum b_{i} e_{i}$, then we have

$$
\alpha(x)^{2} \cdot \alpha(y)^{2}=\alpha(x \cdot y)^{2}+\sum_{j=1}^{8}\left|f_{j}\right|^{2},
$$

where

$$
\begin{aligned}
& f_{1}=+a_{-4} \bar{b}_{-1}+a_{-3} \bar{b}_{+2}-a_{-2} \bar{b}_{+3}+a_{+1} \bar{b}_{-4}, \\
& f_{2}=-a_{-3} \bar{b}_{+1}+a_{-4} \bar{b}_{-2}+a_{-1} \bar{b}_{+3}+a_{+2} \bar{b}_{-4}, \\
& f_{3}=+a_{-2} \bar{b}_{+1}-a_{-1} \bar{b}_{+2}+a_{-4} \bar{b}_{-3}+a_{+3} \bar{b}_{-4}, \\
& f_{4}=+a_{-1} \bar{b}_{-1}+a_{-2} \bar{b}_{-2}+a_{-3} \bar{b}_{-3}-a_{+4} \bar{b}_{-4}, \\
& f_{5}=+a_{+1} \bar{b}_{+1}+a_{+2} \bar{b}_{+2}+a_{+3} \bar{b}_{+3}-a_{-4} \bar{b}_{+4}, \\
& f_{6}=+a_{+2} \bar{b}_{-1}-a_{+1} \bar{b}_{-2}+a_{+4} \bar{b}_{+3}+a_{-3} \bar{b}_{+4}, \\
& f_{7}=-a_{+3} \bar{b}_{-1}+a_{+4} \bar{b}_{+2}+a_{+1} \bar{b}_{-3}+a_{-2} \bar{b}_{+4}, \\
& f_{8}=+a_{+4} \bar{b}_{+1}+a_{+3} \bar{b}_{-2}-a_{+2} \bar{b}_{-3}+a_{-1} \bar{b}_{+4} .
\end{aligned}
$$

This is done by a direct calculation. We don't know a good way to explain this identity.

THEOREM 14.4. - Let $V$ be an octonion algebra over $F$ and define $G=$ $\operatorname{Aut}(V)$. Let $\mathcal{N}^{\prime}$ be the set of hermitian norms on $V$ satisfying (SD) and let $\mathcal{N}$ be the subset of $\mathcal{N}^{\prime}$ consisting of those norm satisfying $(\mathrm{AN})$ as well. We identify $\mathcal{N}^{\prime}$ with $\mathcal{S}(\mathrm{SO}(V, q))$. Then $\mathcal{N}$ is the image of the unique descent map $\mathcal{S}(G) \rightarrow \mathcal{S}(\mathrm{SO}(V, q))$.

Corollary 14.5. - Let $G(\mathbb{R})$ acts on $\mathcal{N}$ by $g . \alpha=\alpha \circ g^{-1}$. For any $\alpha \in \mathcal{N}$, the stabilizer $K_{\alpha}$ of $\alpha$ in $G(\mathbb{R})$ is a maximal compact subgroup of $G(\mathbb{R})$. The correspondence $\alpha \mapsto K_{\alpha}$ is a bijection from $\mathcal{N}$ to the set of maximal compact subgroups of $G(\mathbb{R})$.

Proof. - Again, this is trivial when $F=\mathbb{R}, V$ is non-split, and $G$ is compact. We now assume that $V$ is split. Again we can assume that $V$ is Zorn's model. Let $\alpha_{0}$ be the element of $\mathcal{N}$ given in the proof of Prop. 14.3. We claim that $K_{\alpha_{0}}$ is a maximal compact subgroup of $G(\mathbb{R})$.

The stabilizer $K_{\alpha_{0}}$ is clearly compact. It is easy to see that $K_{\alpha_{0}}$ is the set of real points of a real algebraic group. Therefore, we can compute $\operatorname{dim} K_{\alpha_{0}}$ by computing $\operatorname{dim}\left(\operatorname{Lie} K_{\alpha_{0}}\right)$.

In fact, as a sub-algebra of $\mathfrak{g l}(V)=\operatorname{End}(V)$, the Lie algebra of $G$ consists of those endomorphisms of $V$ whose matrix representations for the basis

BULletin DE LA SOCIÉtÉ MATHÉMATIQUE DE FRANCE 
$\left\{e_{-4}, e_{-3}, e_{-2}, e_{-1}, e_{1}, e_{2}, e_{3}, e_{4}\right\}$ are of the form

$$
\left(\begin{array}{cccccccc}
0 & a^{\prime} & b^{\prime} & c^{\prime} & c & b & a & 0 \\
a & x & p^{\prime} & q^{\prime} & b^{\prime} & -c^{\prime} & 0 & -a \\
b & p & y & r^{\prime} & -a^{\prime} & 0 & c^{\prime} & -b \\
c & q & r & z & 0 & a^{\prime} & -b^{\prime} & -c \\
c^{\prime} & b & -a & 0 & -z & -r^{\prime} & -q^{\prime} & -c^{\prime} \\
b^{\prime} & -c & 0 & a & -r & -y & -p^{\prime} & -b^{\prime} \\
a^{\prime} & 0 & c & -b & -q & -p & -x & -a^{\prime} \\
0 & -a^{\prime} & -b^{\prime} & -c^{\prime} & -c & -b & -a & 0
\end{array}\right)
$$

with $x+y+z=0$. When $F=\mathbb{R}$, Lie $K_{\alpha_{0}}$ consists of real matrices of the above form which are anti-symmetric, so $\operatorname{dim} K_{\alpha_{0}}=6$. When $F=\mathbb{C}$, Lie $K_{\alpha_{0}}$ consists of complex matrices of the above form which are anti-hermitian, so $\operatorname{dim} K_{\alpha_{0}}=14$. Because the maximal compact subgroups of $G(\mathbb{R})$ are connected of dimension 6 when $F=\mathbb{R}$, of dimension 14 when $F=\mathbb{C}, K_{\alpha_{0}}$ must be a maximal compact subgroup.

By Prop. $14.2, G(\mathbb{R})$ acts on the set $\mathcal{N}$ transitively. Therefore, by composing $\alpha \mapsto K_{\alpha}$ with the inverse of $\theta \mapsto K_{\theta}$, we get a bijection $\alpha \mapsto \theta_{\alpha}$ between $\mathcal{N}$ and $\mathcal{S}(G)$. We identify $\mathcal{N}$ with $\mathcal{S}(G)$.

It remains to show that under this identification, the inclusion $\mathcal{N} \subset \mathcal{N}^{\prime}$ is the unique descent map $\mathcal{S}(G) \rightarrow \mathcal{S}(\mathrm{SO}(V, q))$. When $F=\mathbb{C}$, this follows from Prop. 13.8.

When $F=\mathbb{R}$, we consider the following commutative diagram

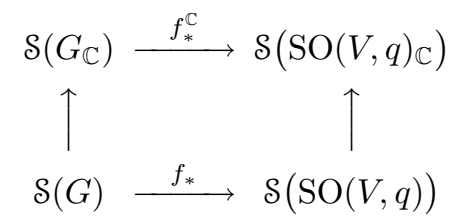

where the horizontal arrows are the unique descent maps, and the vertical arrows are base change maps. Let $y \in \mathcal{S}\left(G_{\mathbb{C}}\right)$ be such that $K_{y} \subset G_{\mathbb{C}}(\mathbb{R})$ is the stabilizer of the norm $\alpha_{\mathbb{C}}$ on $V_{\mathbb{C}}$ constructed in the proof of Prop. 14.3. It is clear that $K_{y}$ is stable under the complex conjugation. By Prop. 13.7, $y$ is the image of some $x \in \mathcal{S}(G)$ under the base change map. By definition, $K_{x}=K_{y} \cap G(\mathbb{R})$. It is easy to see that $K_{x}$ is the stabilizer of $\alpha_{0}=\alpha_{\mathbb{C}} \mid V$.

By Prop. 13.8, $f_{*}^{\mathbb{C}}(y) \in \mathcal{S}\left(\mathrm{SO}(V, q)_{\mathbb{C}}\right) \simeq \mathcal{N}_{\mathbb{C}}^{\prime}$ is simply $\alpha_{\mathbb{C}}$. It is easy to see that $\alpha_{\mathbb{C}}$ is the image of $\alpha_{0} \in \mathcal{S}(\mathrm{SO}(V, q)) \simeq \mathcal{N}^{\prime}$ under the base change map. By the commutativity of the above diagram, we have $f_{*}(x)=\alpha_{0}$. This shows that $f_{*}$ is indeed the inclusion $\mathcal{N} \rightarrow \mathcal{N}^{\prime}$. Now the theorem is proved completely.

We can identify $\mathcal{S}(\operatorname{SO}(V))=\mathcal{S}(\operatorname{Spin}(V))$ with the set $\mathcal{N}^{\prime}$ of self-dual norms on $V$. Under this identification, $\mathbb{Z} / 3 \mathbb{Z}$ acts on $\mathcal{N}^{\prime}$, and the action can be described as follows: let $\sigma$ be a generator of $\mathbb{Z} / 3 \mathbb{Z} \subset \operatorname{Aut}(\operatorname{Spin}(V))$. Each $\alpha \in \mathcal{N}^{\prime}$ defines a Cartan involution $\theta_{\alpha}^{\sharp}$ on $\mathfrak{g l}(V)$ which restricts to a Cartan involution 
$\theta_{\alpha}$ on $\mathfrak{s o}(V)$. The Cartan involution $(\operatorname{Lie} \sigma) \circ \theta_{\alpha} \circ(\operatorname{Lie} \sigma)^{-1}$ is $\theta_{\sigma \cdot \alpha}$. Therefore, the condition $\sigma \cdot \alpha=\alpha$ can be verified using linear algebra computations.

Corollary 14.6. - Let $\alpha \in \mathcal{N}^{\prime}$ be a self-dual norm on $V$. Then $\sigma \cdot \alpha=\alpha$ if and only if $\alpha$ is also an algebra norm for the octonion $V$.

Proof. - This is clear from the preceding theorem, and Thm. 13.11.

The corollary is interesting because as we just explained, the condition $\sigma \cdot \alpha=\alpha$ is easy to verify using linear algebra. However, unlike the $p$-adic case (Lemma 2.1), we do not know a good way of verifying that $\alpha$ is an algebra norm in general.

\section{BIBLIOGRAPHY}

[1] Aschbacher (M.) - Chevalley group of type $G_{2}$ as the group of a trilinear form, J. Algebra, t. 109 (1987), pp. 193-259.

[2] VAn Der BliJ (F.) \& Springer (T.A.) - The arithmetic of octaves and the group $G_{2}$, Indag. Math., t. 21 (1959), pp. 406-418.

[3] Bruhat (F.) \& Tits (J.) - Groupes réductifs sur un corps local I, Publ. Math. IHES, t. 41 (1972), pp. 5-251.

[4] Groupes réductifs sur un corps local II, Publ. Math. IHES, t. 60 (1984), pp. 197-376.

[5] - Schémas en groupes et immeubles des groupes classiques sur un corps local, Bull. Soc. Math. France, t. 112 (1984), pp. 259-301.

[6] - Groupes algébriques sur un corps local, J. Fac. Sci. Univ. Tokyo Sect. IA Math., t. 34 (1987), no. 3, pp. 671-698.

[7] _ Schémas en groupes et immeubles des groupes classiques sur un corps local II: groupes unitaires, Bull. Soc. Math. France, t. 115 (1987), pp. 141-195.

[8] GÉrardin (P.) - Immeubles des groupes linéaires généraux, in Noncommutative harmonic analysis and Lie groups (Marseille, 1980), Lecture Notes in Math., vol. 880, Springer-Verlag, 1981, pp. 138-178.

[9] Grothendieck (A.) \& AL. - SGA 3: Schémas en groupes I, II, III, Lecture Notes in Math., vol. 151, 152, 153, Springer-Verlag, Heidelberg, 1970 .

[10] Knus (M.-A.), Merkurjev (A.), Rost (M.) \& Tignol (J.-P.) - The book of involutions, AMS Colloquium Publ., vol. 44, American Mathematical Society, Providence, RI, 1998.

[11] Landvogt (E.) - Some functorial properties of the Bruhat-Tits building, J. reine ang. Math., t. 518 (2000), pp. 213-241.

[12] Prasad (G.) - Galois-fixed points in the Bruhat-Tits building of a reductive group, Bull. Soc. Math. France, t. 129 (2001), no. 2, pp. 169-174. 
[13] PRAsAd (G.) \& YU (J.-K.) - On finite group actions on reductive groups and buildings, Invent. Math., t. 147 (2002), no. 3, pp. 545-560.

[14] Satake (I.) - Algebraic structures of symmetric domains, Princeton Univ. Press, Princeton, N.J., 1980.

[15] Springer (T.A.) - Reductive groups, in Automorphic forms, representations and L-functions (Borel (A.) \& Casselman (W.), eds.), Proc. Sympos. Pure Math., vol. XXXIII, Amer. Math. Soc., 1979, pp. 3-27.

[16] Linear algebraic groups, 2nd ed., Progress in Math., vol.9, Birkhäuser, Boston, 1998.

[17] Tits (J.) - Reductive groups over local fields, in Automorphic forms, representations and L-functions (Borel (A.) \& Casselman (W.), eds.), Proc. Sympos. Pure Math., vol. XXXIII, Amer. Math. Soc., 1979, pp. 29-69.

[18] YU (J.-K.) - Descent maps of Bruhat-Tits buildings, in preparation.

[19] Smooth models associated to Moy-Prasad groups, in preparation.

[20] _ Minimal K-types of classical groups, preprint, 1998. 